\title{
Response and receptivity of the hypersonic boundary layer past a wedge to free-stream acoustic, vortical and entropy disturbances
}

\author{
Fufeng Qin ${ }^{1} \quad$ Xuesong $\mathrm{Wu}^{1,2}$ \\ ${ }^{1}$ Department of Mechanics, Tianjin University, Tianjin 300072, China \\ ${ }^{2}$ Department of Mathematics, Imperial College London, \\ 180 Queen's Gate, London SW7 2AZ, UK
}

April 19, 2016

\begin{abstract}
This paper analyses the response and receptivity of the hypersonic boundary layer over a wedge to free-stream disturbances including acoustic, vortical and entropy fluctuations. Due to the presence of an attached oblique shock, the boundary layer is known to support viscous instability modes whose eigenfunctions are oscillatory in the far field. These modes acquire a triple-deck structure. Any of three elementary types of disturbances with frequency and wavelength on the triple-deck scales interacts with the shock to generate a slow acoustic perturbation, which is reflected between the shock and the wall. Through this induced acoustic perturbation, vortical and entropy free-stream disturbances drive significant velocity and temperature fluctuations within the boundary layer, which is impossible when the shock is absent. A quasi-resonance was identified, due to which the boundary layer exhibits a strong response to a continuum of high-frequency disturbances within a narrow band of streamwise wavenumbers. Most importantly, in the vicinity of the lower-branch neutral curve the slow acoustic perturbation induced by a disturbance of suitable frequency and wavenumbers is in exact resonance with a neutral eigen mode. As a result, the latter can be generated directly by each of three types of free-stream disturbances without involving any surface roughness element. The amplitude of the instability mode is determined by analysing the disturbance evolution through the resonant region. The fluctuation associated with the eigen mode turns out to be much stronger than free-stream disturbances due to the resonant nature of excitation and in the case of acoustic disturbances, to the well-known amplification effect of a strong shock. Moreover, excitation at the neutral position means that the instability mode grows immediately without undergoing any decay, or missing any portion of the unstable region. All these indicate that this new mechanism is particularly efficient. The boundary-layer response and coupling coefficients are calculated for typical values of parameters.
\end{abstract}

\section{Introduction}

Laminar-turbulent transition of hypersonic boundary layers is of great technological importance for the development of high-speed vehicles (Reshotko 2008) and as such, it has been and still remains a subject of extensive investigation (Fedorov 2011, Zhong \& Wang 2012). Similar to its subsonic counterpart, super- and hypersonic boundary-layer transition involves, in the case of relatively weak free-stream turbulence, four stages in sequence: 
receptivity, linear instability, nonlinear development and final breakdown into small-scale turbulence. Except perhaps linear instability, the rest remain inadequately understood despite substantial efforts in the last few decades.

The first stage, receptivity, refers to the process in which external disturbances in the ambient environment trigger internal oscillations, i.e. eigen modes, in the boundary layer (Morkovin 1969, Reshotko 1976). Instability modes which may arise depend on the Mach number $M$. In the subsonic regime, they are Tollmien-Schlichting (T-S) waves, which have a viscous origin. In the super- and hypersonic regimes, there exist multiple families of modes (Mack 1984) including the so-called first Mack modes, which are continuation of T-S modes into the compressible regime, and the second Mack modes, which are essentially inviscid. On the other hand, external disturbances are present on the wall (such as surface roughness or waviness) and in the incoming flow. Within linear approximation, a general unsteady disturbance in a uniform compressible flow can be decomposed into three independent elementary constituents: acoustic, vorticity and entropy modes (Kovasznay 1953). An acoustic mode corresponds to a pressure wave propagating through the moving fluid at the speed of sound, while vorticity and entropy modes represent respectively weak vorticity and temperature perturbations being convected passively by the mean flow. Usually none of these disturbances alone can excite eigen modes because its length and/or time scales differ from those of the latter. The main task of receptivity study is thus to identify scale-conversion mechanisms which produce an effective forcing with both the time and length scales matching those of the instability.

Breakthroughs towards understanding and predicting boundary-layer receptivity were made in 1980s, when some crucial scale-conversion mechanisms were identified and described mathematically. Goldstein (1983) showed that a long-wavelength acoustic wave can interact with the non-parallel mean flow near the leading edge first to excite the socalled Lam-Rott asymptotic eigen solution, which then undergoes wavelength shortening and finally evolves into an unstable T-S wave. Since the Lam-Rott mode experiences severe decay before reaching the neutral stability point, this mechanism is fairly weak. An efficient mechanism was proposed independently by Ruban (1984) and Goldstein (1985). It involves the interaction between a sound wave and the mean-flow distortion caused by abrupt changes in the wall geometry (such as a hump or a junction of an elliptic leading edge with the straight portion of the plate). The unsteady forcing resulting from the interaction excites a T-S wave provided that the frequency of the sound and the length scale of the abrupt change are comparable with those of the T-S instability. The coupling coefficient was calculated using the triple-deck asymptotic formalism.

Duck, Ruban \& Zhikharev (1996) considered the receptivity due to free-stream vortical disturbances interacting with a local roughness on a flat plate. They pointed out that although a vortical disturbance does not penetrate into the boundary layer, it can interact with the roughness-induced steady perturbation at the outer edge of the boundary layer. $\mathrm{Wu}(2001 \mathrm{a})$ presented a second-order asymptotic theory and demonstrated that irrespective of the vertical structure of the vortical disturbance, the receptivity can be fully characterized by its slip velocity at the edge of the boundary layer. This allowed for quantitative comparisons with the experimental data of Dietz (1999), and an excellent agreement was found. Wu (2001b) investigated the distributed receptivity due to acoustic or vortical disturbances interacting with a wavy wall. The forcing resonates with a neutral T-S mode in a fairly extended vicinity of the lower-branch neutral curve, and as a result the receptivity is much stronger than in the case of an isolated roughness.

A rather different mechanism of generating T-S waves was proposed by Wu (1999). It 
involves the interaction between a vortical disturbance and a sound wave with suitable frequencies and wavenumbers to generate a forcing in resonance with a neutral mode. Since this mechanism does not resort to any form of surface inhomogeneity, it operates effectively in canonical boundary layers over smooth flat walls.

For supersonic boundary layers, possible instability modes have been identified and their characteristics, obtained by solving compressible Orr-Sommerfeld and Rayleigh equations, have been well documented (Mack 1984). Relevant asymptotic descriptions have been presented by Smith (1989), Smith \& Brown (1990) and Blackby, Cowley \& Hall (1993) among others. Most calculations and theories neglected shocks, which are present when the aerodynamic body has a finite thickness. In the hypersonic regime, a shock arises even in a flat-plat boundary layer because of the strong viscous-inviscid interaction near the leading edge. The presence of a shock could influence instability as perturbations may be reflected between the shock and boundary, and this effect can, as was first recognized by Petrov (1984), be accounted for by imposing the linearized Rankine-Hugoniot relations as boundary conditions on the linearized stability equations. Adopting these conditions and the high-Reynolds-number asymptotic framework of triple-deck formalism, Cowley \& Hall (1990) analysed the stability of the boundary layer over a sharp wedge. They found that the presence of a shock gives rise to an infinite but countable set of unstable viscous (i.e. T-S) modes, whose eigenfunctions are oscillatory in the far field (see also Seddougui \& Bassom 1994). Chang, Malik \& Hussaini (1990) performed finite-Reynolds-number linear stability calculations under the parallel-flow assumption. They found that a shock located well outside the boundary layer has little influence on stability, whereas a shock located near the edge of the boundary layer stabilizes the first and second Mack modes, but also induces additional supersonic modes whose eigenfunctions are oscillatory between the shock and boundary-layer edge, consistent with the prediction by the asymptotic theory.

Experiments indicate that transition in the supersonic regime is significantly affected by free-stream disturbances (Laufer 1954, Kendall 1975, Graziosi \& Brown 2002) and wall roughness (e.g. Patel 1971). In laboratory conditions, acoustic noise emitted from the turbulent boundary layers on the tunnel walls has a strong impact (Laufer 1960, Schneider 2001), and the transition front in quiet and conventional tunnels can be drastically different (King 1992). All these observations testify the crucial role of receptivity. However, there have been just a few detailed measurements of receptivity using controlled free-stream disturbances, and these were conducted by Maslov et al. (2001) and Semionov \& Kosinov $(1999,2008)$ for flat plates with sharp and blunt leading edges.

Receptivity of supersonic boundary layers becomes more complex than its subsonic counterpart because, inter alia, there exist multiple families of eigen modes, among which one may convert to another at certain locations of the flow. For reviews of the subject, the reader is referred to Fedorov (2011) and Zhong \& Wang (2012). In order to understand receptivity to free-stream disturbances, Fedorov \& Khokhlov $(1991,2001)$ examined the limiting behaviour of boundary-layer instability modes in the leading-edge region, and found that there exist so-called slow and fast modes, whose phase speeds (normalized by the free-stream velocity) approach $(1-1 / M)$ and $(1+1 / M)$ respectively. These modes are thus almost synchronized respectively with slow and fast sound waves propagating in the free stream. A slow mode develops into a first Mack mode. Fedorov \& Khokhlov (1991, 2001) and Fedorov (2003) then considered coupling of slow and fast modes with sound waves being incident upon the boundary layer at different angles $\theta$. When $\theta$ is sufficiently small, only the slow mode is excited, by the refracted sound wave. When $\theta=O(1)$, the incident wave is scattered by the leading edge, generating both slow and fast sound 
waves, which in turn excite the fast and slow boundary-layer modes respectively. The phase speed of the fast mode decreases with the downstream distance and becomes equal to that of a second Mack mode, at which point (referred to as synchronization point) inter-modal exchange occurs, that is, the fast mode converts into an amplifying second Mack mode (Fedorov \& Khokhlov 2001). Unlike Lam-Rott asymptotic eigen modes, slow modes do not experience server decay, and hence the present receptivity to slow acoustic waves is much more efficient than the leading-edge adjustment mechanism in the subsonic case (Goldstein 1983). Fast modes do undergo decay and hence the receptivity to fast sound waves should be relatively weaker. Fedorov (2003) made detailed comparison with the experimental data of Maslov et al. (2001) and observed reasonably good agreement for planar or moderately three-dimensional disturbances, but the prediction deteriorates and even exhibits opposite trends when three-dimensionality becomes sufficiently strong. Indeed, highly oblique instability modes with spanwise wavenumbers $\beta>\sqrt{M^{2}-1} \alpha$ have phase speeds much smaller than the free-stream velocity and acquire a triple-deck structure (Smith 1989), where $\alpha$ is the streamwise wavenumber. The asymptotic theory of Fedorov \& Khokhlov (2001) ceases to be valid for these modes.

Direct numerical simulations (DNS) of receptivity of a flat-plat boundary layer were conducted by Ma \& Zhong (2003a,b, 2005) and Balakumar (2003, 2005). These simulations, in which shocks are present and accounted for, show that in the presence of free-stream acoustic, vortical and entropy disturbances, first and second modes are excited. The boundary layer responds directly to slow and fast acoustic disturbances in the leading-edge region as is described by the theory of Fedorov (2003) with the receptivity to slow sound waves being stronger. Vortical and entropy perturbations excite instability modes indirectly, namely, they first interact with the shock to generate acoustic fluctuations behind the shock, which in turn excite slow and fast boundary-layer modes near the leading edge, presumably through the mechanism of Fedorov \& Khokhlov (1991) and Fedorov (2003). Generation of second Mack modes involves two steps: excitation of fast or slow modes and the intermodal conversion at the synchronization point as explained by Fedorov \& Khokhlov (2001). Simulations for receptivity of boundary layers over parabola, blunt plates and cones, carried out by Zhong (2001), Zhong \& Ma (2006) and Balakumar (2009), indicate that bluntness reduces receptivity as was suggested by theoretical analysis (Egorov, Fedorov \& Nechaev 2004). Interestingly, in the case of parabola, the receptivity is stronger to fast acoustic waves than to slow ones (Zhong 2001), while the opposite is true for sharp wedges or cones (Balakumar \& Kegerise 2010).

For supersonic/hypersonic boundary layers, shocks may play a crucial role in the receptivity to free-stream disturbances. As is well known, when each of three elementary types of free-stream disturbances strikes a shock in free space, all three types of waves may emerge downstream (McKenzie \& Westphal 1968). A similar process takes place when a shock is adjacent to a solid boundary, as was shown theoretically by Duck, Lasseigne \& Hussaini $(1995,1997)$ for the supersonic flow past a wedge, and demonstrated experimentally by Maslov et al. (2010) for the flat-plat boundary layer in the strongly interactive regime. In the presence of the boundary layer, the disturbance downstream also consists of reflected acoustic waves. The acoustic waves downstream the shock penetrate into the boundary layer to drive substantial response there. In this paper, we consider the boundary layer over a sharp wedge, and investigate possible generation of instability waves by these waves. We will show that for disturbances with appropriate time and length scales the resultant slow acoustic waves may be in resonance with neutral T-S waves near the lower branch of the neutral curve. As a result, a T-S wave can be excited directly by any 
of acoustic (slow or fast), vortical and entropy disturbances in the free stream. This is a completely new mechanism, which involves neither interaction with a surface roughness, nor interaction between free-stream disturbances themselves.

The rest of the paper is organised as follows. In $\S 2$, we formulate the problem. The base flow, including the inviscid field and the viscous boundary layer, is briefly described. The unsteady disturbances in the oncoming flow are specified. Their interaction with an oblique shock and the resulting shock conditions are highlighted. We specify the time and length scales of the disturbances such that the boundary-layer response is viscous, and more importantly the forcing may resonate with a T-S mode near the lower branch of the neutral curve. The scalings lead to a triple-deck structure governing the induced disturbance. In $\S 3$, the boundary-layer response in the pre-resonance region upstream of the neutral curve is analysed. The resonance between the forcing and the neutral mode is considered in $\S 4$. In $\S 5$, by matching the solutions in the pre-resonance and resonance regions, we determine the initial amplitude of the T-S wave excited. Appropriate coupling coefficients for three types of free-stream disturbances are then defined. Numerical results for the boundary-layer response in the pre-resonance region and for the coupling coefficients of the receptivity will be presented in $\S 6$. The results are summarised and discussed in $\S 7$.

\section{Formulation}

We consider a supersonic flow past a wedge with a semi angle $\theta$. The wedge is taken to be symmetrically aligned with the oncoming flow. A straight shock forms and makes an acute angle $\sigma$ with the symmetry line of the wedge as is illustrated in figure 1; the angle between the shock and the wedge surface is $\phi=\sigma-\theta$. The steady velocity, density, pressure and temperature are denoted by $\breve{u}, \breve{\rho}, \breve{p}$ and $\breve{T}$ respectively. Quantities upstream of the shock are indicated by the subscript ' $u$ ', whereas those between the shock and wedge by the subscript ' $s$ '. The oncoming flow has a mean speed $\breve{U}_{u}$, and is perturbed by threedimensional small-amplitude disturbances, which are to be specified shortly. The fluid is assumed to be a perfect gas with a constant ratio of specific heats $\gamma$. Based upon $\breve{U}_{u}$ and the upstream sound speed $\breve{a}_{u}=\sqrt{\gamma \breve{p}_{u} / \breve{\rho}_{u}}$, the upstream Mach number $M_{u}$ is defined as

$$
M_{u}=\breve{U}_{u} / \breve{a}_{u} .
$$

The uniform velocity, density, pressure, temperature, shear viscosity and sound speed between the shock and the wedge are denoted by $\breve{U}_{s}, \breve{\rho}_{s}, \breve{p}_{s}, \breve{T}_{s}, \breve{\mu}_{s}$ and $\breve{a}_{s}$ respectively. Using these quantities, we define the Reynolds number $R e$ and the Mach number $M$ as

$$
R e=\breve{\rho}_{s} \breve{U}_{s} \breve{l} / \breve{\mu}_{s}, \quad M=\breve{U}_{s} / \breve{a}_{s},
$$

where $\breve{l}$ denotes the distance from the wedge tip to the location of interest.

The inviscid solution for the steady flow relates the uniform quantities upstream and downstream the shock (e.g. see Hayes \& Probstein 2004). Specifically,

$$
\begin{gathered}
\epsilon \equiv \frac{\breve{\rho}_{u}}{\breve{\rho}_{s}}=\left(\frac{\gamma-1}{\gamma+1}\right)\left(1+\frac{2}{(\gamma-1) M_{u}^{2} \sin ^{2} \sigma}\right), \quad \frac{\breve{p}_{s}}{\breve{p}_{u}}=1+\gamma M_{u}^{2} \sin ^{2} \sigma(1-\epsilon) \\
\tan \phi=\epsilon \tan \sigma, \quad M^{2}=\frac{M_{u}^{2} \cos ^{2} \sigma\left(1+\epsilon^{2} \tan ^{2} \sigma\right)}{1+\frac{1}{2}(\gamma-1)\left(1-\epsilon^{2}\right) M_{u}^{2} \sin ^{2} \sigma} .
\end{gathered}
$$

Two Cartesian coordinate systems will be used to describe different aspects of the flow. The interaction of unsteady disturbances with the shock will be analysed in the system 


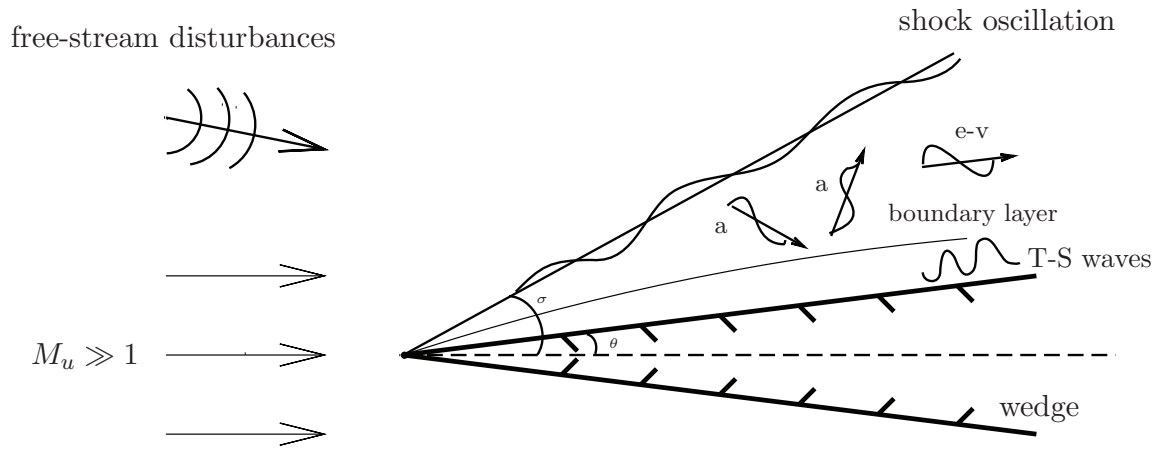

Figure 1: Flow configuration and unsteady disturbances. The letters ' $a$ ', ' $v$ ' and 'e' refer to acoustic, vortical and entropy perturbations respectively.

oriented with the shock. The boundary-layer flow will be considered in the system $(x, y, z)$ with its origin at the wedge tip, where $x$ and $y$ are along and normal to the wedge surface respectively, and $z$ is in the spanwise direction, all non-dimensionalized by $\breve{l}$. The time variable $t$ is normalized by $\breve{l} / \breve{U}_{s}$. The velocity $(u, v, w)$, density $\rho$, pressure $p$, temperature $T$, and shear and bulk viscosities $\mu$ and $\mu^{\prime}$ are non-dimensionalized by $\breve{U}_{s}, \breve{\rho}_{s}, \breve{\rho}_{s} \breve{U}_{s}^{2}, \breve{T}_{s}$ and $\breve{\mu}_{s}$ respectively. The flow is governed by the compressible Navier-Stokes (N-S) equations,

$$
\begin{gathered}
\frac{\partial \rho}{\partial t}+\nabla \cdot(\rho \boldsymbol{u})=0, \\
\rho \frac{\mathrm{D} \boldsymbol{u}}{\mathrm{D} t}=-\nabla p+\frac{1}{R e}\left[\nabla \cdot(2 \mu \boldsymbol{e})+\nabla\left(\left(\mu^{\prime}-\frac{2}{3} \mu\right) \nabla \cdot \boldsymbol{u}\right)\right], \\
\rho \frac{\mathrm{D} T}{\mathrm{D} t}=(\gamma-1) M^{2} \frac{\mathrm{D} p}{\mathrm{D} t}+\frac{1}{\operatorname{Pr} R e} \nabla \cdot(\mu \nabla T)+\frac{(\gamma-1) M^{2}}{\operatorname{Re}} \Phi, \\
\gamma M^{2} p=\rho T,
\end{gathered}
$$

where $\boldsymbol{e}$ and $\Phi$ denote the strain-rate tensor and the dissipation function respectively,

$$
e_{i j}=\frac{1}{2}\left(\frac{\partial u_{i}}{\partial x_{j}}+\frac{\partial u_{j}}{\partial x_{i}}\right), \quad \Phi=2 \mu \boldsymbol{e}: \boldsymbol{e}+\left(\mu^{\prime}-\frac{2}{3} \mu\right)(\nabla \cdot \boldsymbol{u})^{2},
$$

and $P r$ is the Prandtl number. The dependence of the shear viscosity $\mu$ on the temperature is described by a viscosity law. For the present problem, the viscosity law does not affect the result qualitatively. For simplicity, we use the Chapman law, which can be written as $\mu=T$ for the normalization adapted. Furthermore we take $\mu^{\prime}=0$.

\subsection{The base flow}

In the coordinate system $(\xi, \eta, z)$, the inviscid part of the base flow is given by (Cowley \& Hall 1990)

$$
\begin{gathered}
\bar{R}=\epsilon, \quad R=1, \quad \bar{U}=U=\frac{1}{\left(1+\epsilon^{2} \mathscr{T}^{2}\right)^{\frac{1}{2}}}, \quad \bar{V}=-\frac{\mathscr{T}}{\left(1+\epsilon^{2} \mathscr{T}^{2}\right)^{\frac{1}{2}}}, \quad V=\epsilon \bar{V}, \\
\bar{W}=W=0, \quad \bar{P}=\frac{\epsilon}{\gamma M^{2}}-\frac{\epsilon(\gamma-1)\left(1-\epsilon^{2}\right) \mathscr{T}^{2}}{2 \gamma\left(1+\epsilon^{2} \mathscr{T}^{2}\right)}, \quad P=\frac{1}{\gamma M^{2}}, \quad \mathscr{T}=\tan \sigma, \\
\bar{E}=\frac{\bar{P}}{(\gamma-1) \bar{R}}+\frac{1}{2}\left(\bar{U}^{2}+\bar{V}^{2}\right), \quad E=\frac{P}{(\gamma-1) R}+\frac{1}{2}\left(U^{2}+V^{2}\right),
\end{gathered}
$$


where a quantity with/without a bar indicates its value upstream/downstream the shock.

The viscous boundary layer is described by the transverse variable

$$
\tilde{Y}=R e^{\frac{1}{2}} y
$$

and its streamwise velocity profile is given by the similarity solution

$$
U_{B}=f^{\prime}(\zeta) \text { with } \zeta=\frac{1}{\sqrt{x}} \int_{0}^{\tilde{Y}} R_{B} \mathrm{~d} \tilde{Y}
$$

where $R_{B}$ in $\zeta$ (the Dorodnitsyn-Howarth variable) is the mean density profile. It can be shown that

$$
U_{B} \rightarrow \lambda \mu_{w}^{-\frac{1}{2}} T_{w}^{-\frac{1}{2}} \tilde{Y} \text { as } \tilde{Y} \rightarrow 0,
$$

where $\lambda=\chi x^{-\frac{1}{2}}$ with $\chi \approx 0.332$, while $\mu_{w}$ and $T_{w}$ denote the shear viscosity and temperature on the wall respectively; for the Chapman law, $\mu_{w}=T_{w}$. For simplicity, we assume that (a) the wedge walls are insulating, i.e. $\partial T_{B} / \partial \tilde{Y}=0$ on $\tilde{Y}=0$, and (b) the Prandtl number is unity, i.e. $\operatorname{Pr}=1$. Then the temperature and density of the boundary-layer flow, $T_{B}$ and $R_{B}$, are given by

$$
T_{B}=1+\frac{\gamma-1}{2} M^{2}\left(1-U_{B}^{2}\right), \quad R_{B}=T_{B}^{-1} .
$$

\subsection{Unsteady disturbances}

Upstream of the shock, an unsteady perturbation with a small amplitude of order $\delta \ll 1$ is superimposed on the steady flow. In order to derive the so-called shock condition satisfied by the disturbance downstream of the shock, it is convenient to work with the coordinate system $(\xi, \eta, z)$, where $\xi, \eta$ are parallel and normal to the shock respectively, and $z$ is in the spanwise direction; they are normalized by $\breve{l}$, the distance to the leading edge. The corresponding velocity field is denoted by $(u, v, w)$. The total flow field can be written as

$$
(\rho, u, v, w, p, \mathscr{E})=(\bar{R}, \bar{U}, \bar{V}, 0, \bar{P}, \bar{E})+\delta\left(\tilde{r}_{0}, \tilde{u}_{0}, \tilde{v}_{0}, \tilde{w}_{0}, \tilde{p}_{0}, \tilde{e}_{0}\right),
$$

where $\mathscr{E}=p /[(\gamma-1) \rho]+\left(u^{2}+v^{2}+w^{2}\right) / 2$.

As the background flow is uniform, the perturbation can be decomposed as

$$
\begin{gathered}
\tilde{e}_{0}=\frac{1}{(\gamma-1) \bar{R}} \tilde{p}_{0}-\frac{\bar{P}}{(\gamma-1) \bar{R}^{2}} \tilde{r}_{0}+\bar{U} \tilde{u}_{0}+\bar{V} \tilde{v}_{0}, \\
\tilde{u}_{0}=\tilde{u}_{0 a}^{+}+\tilde{u}_{0 a}^{-}+\tilde{u}_{0 v}, \quad \tilde{v}_{0}=\tilde{v}_{0 a}^{+}+\tilde{v}_{0 a}^{-}+\tilde{v}_{0 v}, \quad \tilde{w}_{0}=\tilde{w}_{0 a}^{+}+\tilde{w}_{0 a}^{-}+\tilde{w}_{0 v}, \\
\tilde{p}_{0}=\tilde{p}_{0 a}^{+}+\tilde{p}_{0 a}^{-}, \quad \tilde{r}_{0}=\tilde{r}_{0 a}^{+}+\tilde{r}_{0 a}^{-}+\tilde{r}_{0 e},
\end{gathered}
$$

where the subscripts ' $a$ ', ' $v$ ' and ' $e$ ' indicate acoustic, vorticity and entropy modes respectively with the sign ' $+/-$ ' referring to fast/slow acoustic waves respectively.

Each of three types of perturbations is governed by the linearized N-S equations about the uniform mean field. Acoustic waves are irrotational, and their pressure, density and velocity are found as

$$
\left.\begin{array}{l}
\tilde{p}_{0 a}^{ \pm}=p_{I}^{ \pm} \mathrm{e}^{\mathrm{i} \nu_{0}^{ \pm} \eta} \tilde{E}_{0}+\text { c.c., } \quad \tilde{r}_{0 a}^{ \pm}=M_{u}^{2} \frac{\cos ^{2} \sigma}{\cos ^{2} \phi} p_{I}^{ \pm} \mathrm{e}^{\mathrm{i} \nu_{0}^{ \pm} \eta} \tilde{E}_{0}+\text { c.c. }, \\
\left(\tilde{u}_{0 a}^{ \pm}, \tilde{v}_{0 a}^{ \pm}, \tilde{w}_{0 a}^{ \pm}\right)=\left(\alpha, \nu_{0}^{ \pm}, \beta\right) \frac{p_{I}^{ \pm}}{\left(\omega-\alpha \bar{U}-\nu_{0}^{ \pm} \bar{V}\right) \bar{R}} \mathrm{e}^{\mathrm{i} \nu_{0}^{ \pm} \eta} \tilde{E}_{0}+\text { c.c. },
\end{array}\right\}
$$


where we have introduced

$$
\tilde{E}_{0}=\mathrm{e}^{\mathrm{i}(\alpha \xi+\beta z-\omega t)},
$$

and the frequency and wavenumbers satisfy the acoustic dispersion relation

$$
\omega-\alpha \bar{U}-\nu_{0}^{ \pm} \bar{V}= \pm \frac{\cos \phi}{M_{u} \cos \sigma} \sqrt{\alpha^{2}+\left(\nu_{0}^{ \pm}\right)^{2}+\beta^{2}} .
$$

A vorticity wave has, on the other hand, no pressure fluctuation, and its velocity components take the form

$$
\left(\tilde{u}_{0 v}, \tilde{v}_{0 v}, \tilde{w}_{0 v}\right)=\left(u_{I}, v_{I}, w_{I}\right) \mathrm{e}^{\mathrm{i} \nu_{I} \eta} \tilde{E}_{0}+\text { c.c., }
$$

whereas an entropy mode carries merely a density (or temperature) fluctuation,

$$
\tilde{r}_{0 e}=r_{I} \mathrm{e}^{\mathrm{i} \nu_{I} \eta} \tilde{E}_{0}+\text { c.c. } .
$$

For both vorticity and entropy modes, the wavenumber in the $\eta$-direction, $\nu_{I}$, satisfies the dispersion relation

$$
\omega-\alpha \bar{U}-\nu_{I} \bar{V}=0 .
$$

In general, whenever any one of acoustic, vorticity and entropy waves strikes a shock, all three types of fluctuations may arise altogether downstream. They will be referred to as transmitted waves if they are of the same type as the upstream ones, or generated waves if different. The generated/transmitted waves have the same frequency and the same wavelengths parallel to the shock as the disturbance upstream. For a shock in free space, the disturbances downstream all are transmitted from, and/or generated by, the upstream perturbation. However, for a shock adjacent to a wall, along which a boundary layer develops, there may exist also the free-stream signature of boundary-layer instability modes. This is the case for the present problem. We shall assume that the perturbation has a short wavelength, i.e. $\alpha, \beta \gg 1$. The perturbation downstream the shock is allowed to modulate slowly over a length scale much longer than $2 \pi / \alpha$ but much shorter than $O(1)$. The modulation is described by the variable $x_{1}=x / \tilde{\epsilon}$, where $2 \pi / \alpha \leq \tilde{\epsilon}^{2} \ll 1$. With the above considerations, we write the downstream flow field as

$$
\begin{gathered}
(\rho, u, v, w, p, \mathscr{E})=(R, U, V, 0, P, E)+\delta \tilde{\epsilon}^{-1}\left[\left(\tilde{r}_{1}, \tilde{u}_{1}, \tilde{v}_{1}, \tilde{w}_{1}, \tilde{p}_{1}, \tilde{e}_{1}\right)\right. \\
\left.+\tilde{\epsilon}^{(}\left(\tilde{r}_{2}, \tilde{u}_{2}, \tilde{v}_{2}, \tilde{w}_{2}, \tilde{p}_{2}, \tilde{e}_{2}\right)\right],
\end{gathered}
$$

where the $O(\delta / \tilde{\epsilon})$ term represents the signature of the T-S wave that may be generated by the $O(\delta)$ disturbance in the oncoming flow. The larger magnitude is due to the resonant nature of the forcing. This term is present in the expansion near the neutral position of the T-S wave, but would be absent upstream of the neutral position. The disturbance in (2.17) again consists of acoustic, vortical and entropy parts, namely

$$
\begin{gathered}
\tilde{e}_{j}=\frac{1}{(\gamma-1) R} \tilde{p}_{j}-\frac{P}{(\gamma-1) R^{2}} \tilde{r}_{j}+U \tilde{u}_{j}+V \tilde{v}_{j}, \\
\tilde{u}_{j}=\tilde{u}_{j a}+\tilde{u}_{j v}, \quad \tilde{v}_{j}=\tilde{v}_{j a}+\tilde{v}_{j v}, \quad \tilde{w}_{j}=\tilde{w}_{j a}+\tilde{w}_{j v}, \\
\tilde{p}_{j}=\tilde{p}_{j a}, \quad \tilde{r}_{j}=\tilde{r}_{j a}+\tilde{r}_{j e}, \quad(j=1,2) .
\end{gathered}
$$


The leading-order (i.e. $\left.O\left(\delta \tilde{\epsilon}^{-1}\right)\right)$ acoustic part admits the solution for the pressure

$$
\tilde{p}_{1 a}=\left(\check{a}_{I} \mathrm{e}^{\mathrm{i} \nu_{1} \eta}+\check{a}_{R} \mathrm{e}^{\mathrm{i} \nu_{2} \eta}\right) \tilde{E}_{0},
$$

where $\nu_{1}$ and $\nu_{2}$ are the wavenumbers of the incoming and outgoing slow acoustic waves, and they satisfy the dispersion relation

$$
\omega-\alpha U-\nu_{1,2} V=-\frac{1}{M} \sqrt{\alpha^{2}+\nu_{1,2}^{2}+\beta^{2}},
$$

and the amplitudes of these two waves, $\check{a}_{I}$ and $\check{a}_{R}$, are functions of $x_{1}$. Note that a minus sign on the right-hand-side of (2.20) is taken because a T-S instability mode has a small phase speed, and behaves outside the boundary layer like a slow acoustic wave. The corresponding expressions for the velocities and the solution for the induced vortical part of the disturbance are given in Appendix A. The result will be used in the main text specifically for $\alpha=O\left(R e^{\frac{3}{8}} \mu_{w}^{-\frac{3}{8}} T_{w}^{-\frac{9}{8}} M^{-\frac{3}{4}}\right), \beta=O\left(R e^{\frac{3}{8}} \mu_{w}^{-\frac{3}{8}} T_{w}^{-\frac{9}{8}} M^{\frac{1}{4}}\right)$ and $\tilde{\epsilon}=R e^{-\frac{3}{16}} \mu_{w}^{\frac{3}{16}} T_{w}^{\frac{9}{16}} M^{\frac{3}{8}}$.

The $O(\delta)$ disturbance downstream the shock consists of two parts: (a) that transmitted from, or generated by, the upstream perturbation, and (b) that arising from the modulation of the leading-order (i.e. $O(\delta / \tilde{\epsilon})$ ) fluctuations associated with the T-S mode. The solution for the acoustic part, which is governed by the inhomogeneous wave equation, is of the form

$$
\tilde{p}_{2 a}=\left(\hat{a}_{I} \mathrm{e}^{\mathrm{i} \nu_{1} \eta}+\hat{a}_{R} \mathrm{e}^{\mathrm{i} \nu_{2} \eta}+\hat{p}_{2 a, p}\right) \tilde{E}_{0},
$$

where $\hat{p}_{2 a, p}$ denotes the particular solution, and $\hat{a}_{I}$ and $\hat{a}_{R}$ are functions of $x_{1}$.

For an oblique shock in free space, the relations satisfied by the disturbance on the shock were given by Mckenzie \& Westphal (1968). In the presence of a wall, the analysis must allow for the acoustic wave reflected by the wall as well as for the signature of the boundary-layer perturbations. These were considered by Cowley \& Hall (1990) but the upstream disturbance was assumed absent. Here, we consider the case where the upstream disturbance may be comprised of any of three types of modes. Furthermore, the disturbance downstream may be spatially modulated. The detailed analysis of their interaction with a general oblique shock is performed by Qin \& Wu (2016) for any $\tilde{\epsilon}$ satisfying $2 \pi / \alpha \leq \tilde{\epsilon}^{2} \ll 1$, and the generalised shock conditions are derived. In Appendix A, these conditions are specialised to (2.5a)-(2.5c), leading to (A.7) and (A.20), which relate $\check{a}_{I}$ and $\check{a}_{R}$ in (2.19), and $\hat{a}_{I}$ and $\hat{a}_{R}$ in (2.21), respectively. The relations (A.7) and (A.20) simplify significantly for disturbances on the triple-deck scales.

In the coordinate system $(x, y, z)$, the leading-order pressure $\tilde{p}$ is governed by

$$
M^{2}\left(\frac{\partial}{\partial t}+\frac{\partial}{\partial x}\right)^{2} \tilde{p}-\nabla^{2} \tilde{p}=0 .
$$

The solution takes the form

$$
\tilde{p}=\delta\left[a_{I} \mathrm{e}^{\mathrm{i}\left(\alpha_{a} x+\gamma_{a} y+\beta_{a} z-\omega_{a} t\right)}+a_{R} \mathrm{e}^{\mathrm{i}\left(\alpha_{a} x-\gamma_{a} y+\beta_{a} z-\omega_{a} t\right)}\right] .
$$

Substitution of (2.23) into (2.22) leads to the dispersion relation for slow acoustic waves,

$$
\omega_{a} / \alpha_{a}=1-\frac{1}{M} \sqrt{1+\left(\beta_{a} / \alpha_{a}\right)^{2}+\left(\gamma_{a} / \alpha_{a}\right)^{2}} .
$$

Note that the two coordinate systems, $(x, y, z)$ and $(\xi, \eta, z)$, are related to each other by

$$
x=\xi \cos \phi-\eta \sin \phi, \quad y=\xi \sin \phi+\eta \cos \phi .
$$


The wavenumbers in the two systems are related by

$$
\alpha_{a}=\alpha \cos \phi-\nu_{1,2} \sin \phi, \quad \gamma_{a}=\alpha \sin \phi+\nu_{1,2} \cos \phi,
$$

with which the dispersion relations (2.20) and (2.24) are equivalent as expected. When $\phi \ll 1$, which is the case for our problem, the expressions (2.19) and (2.23) are identical on the shock $(\eta=0)$ provided that

$$
\check{a}_{I}=a_{I} \mathrm{e}^{\mathrm{i} \gamma_{a} y_{s}}, \quad \check{a}_{R}=a_{R} \mathrm{e}^{-\mathrm{i} \gamma_{a} y_{s}},
$$

where $y_{s}=\xi \sin \phi=x \tan \phi$ is the local shock position.

\section{$2.3 \quad$ Asymptotic scalings}

In order to develop an asymptotic theory for receptivity, we assume the Reynolds and Mach numbers to be large: $R e \gg 1$ and $M \gg 1$. More specifically, for the base flow we adopt the same scaling restrictions as those in Cowley \& Hall (1990),

$$
\sigma \ll 1, \quad M \gg 1, \quad \sigma M \gg 1, \quad \epsilon \approx 1 /\left(\sigma^{2} M^{2}\right), \quad \gamma-1 \sim \bar{\gamma} /\left(\sigma^{2} M^{2}\right),
$$

to ensure that the shock is sufficiently close to the wall to influence the boundary-layer instability. Use of (2.28) to the first equation in (2.2) shows that

$$
M_{u}^{2}=\frac{2}{\sin ^{2} \sigma[(\gamma+1) \epsilon-\gamma+1]} \sim \frac{2}{2-\bar{\gamma}} M^{2},
$$

where $\bar{\gamma}$ is an $O(1)$ constant and $0<\bar{\gamma}<2$.

In the limit of $R e \gg 1$ and $M \gg 1$, the characteristic frequency of lower-branch T-S waves, $\omega_{\mathrm{TS}}$, and their wavenumbers in the $x$ - and $z$-directions, $\alpha_{\mathrm{TS}}$ and $\beta_{\mathrm{TS}}$, scale as (Smith 1989; Cowley \& Hall 1990)

$$
\omega_{\mathrm{TS}}=R e^{\frac{1}{4}} \mu_{w}^{-\frac{1}{4}} T_{w}^{-\frac{3}{4}} M^{-\frac{1}{2}} \hat{\omega}_{\mathrm{TS}}, \quad\left(\alpha_{\mathrm{TS}}, \beta_{\mathrm{TS}}\right)=R e^{\frac{3}{8}} \mu_{w}^{-\frac{3}{8}} T_{w}^{-\frac{9}{8}} M^{-\frac{3}{4}}\left(\hat{\alpha}_{\mathrm{TS}}, M \hat{\beta}_{\mathrm{TS}}\right) .
$$

The disturbance in the oncoming free stream is in general of broadband nature and so are those emerging downstream the shock, among which each acoustic wave is characterized by its frequency $\omega_{a}$, and streamwise and spanwise wavenumbers, $\alpha_{a}$ and $\beta_{a}$ (with $\gamma_{a}$ determined by the dispersion relation (2.24)). Our interest is in Fourier components which generate T-S waves. This requires $\omega_{a}, \alpha_{a}$ and $\beta_{a}$ of slow acoustic waves to be on the same triple-deck scales,

$$
\omega_{a}=R e^{\frac{1}{4}} \mu_{w}^{-\frac{1}{4}} T_{w}^{-\frac{3}{4}} M^{-\frac{1}{2}} \hat{\omega}_{a}, \quad\left(\alpha_{a}, \beta_{a}\right)=R e^{\frac{3}{8}} \mu_{w}^{-\frac{3}{8}} T_{w}^{-\frac{9}{8}} M^{-\frac{3}{4}}\left(\hat{\alpha}_{a}, M \hat{\beta}_{a}\right) .
$$

Substitution of the scalings (2.31) into (2.24) leads to

$$
1-\frac{1}{M} \sqrt{1+\left(\hat{\beta}_{a} / \hat{\alpha}_{a}\right)^{2} M^{2}+\left(\gamma_{a} / \alpha_{a}\right)^{2}}=R e^{-\frac{1}{8}} \mu_{w}^{\frac{1}{8}} T_{w}^{\frac{3}{8}} M^{\frac{1}{4}}\left(\hat{\omega}_{a} / \hat{\alpha}_{a}\right) .
$$

In order for the left-hand side of the equation to be positive and asymptotically small, we must require that $\hat{\alpha}_{a}>\hat{\beta}_{a}$ and $\gamma_{a}=\operatorname{Re}^{\frac{3}{8}} \mu_{w}^{-\frac{3}{8}} T_{w}^{-\frac{9}{8}} M^{\frac{1}{4}} \hat{\gamma}_{a}$ with $\hat{\gamma}_{a}$ being given, to leading order, by

$$
\hat{\gamma}_{a}=\sqrt{\hat{\alpha}_{a}^{2}-\hat{\beta}_{a}^{2}}
$$




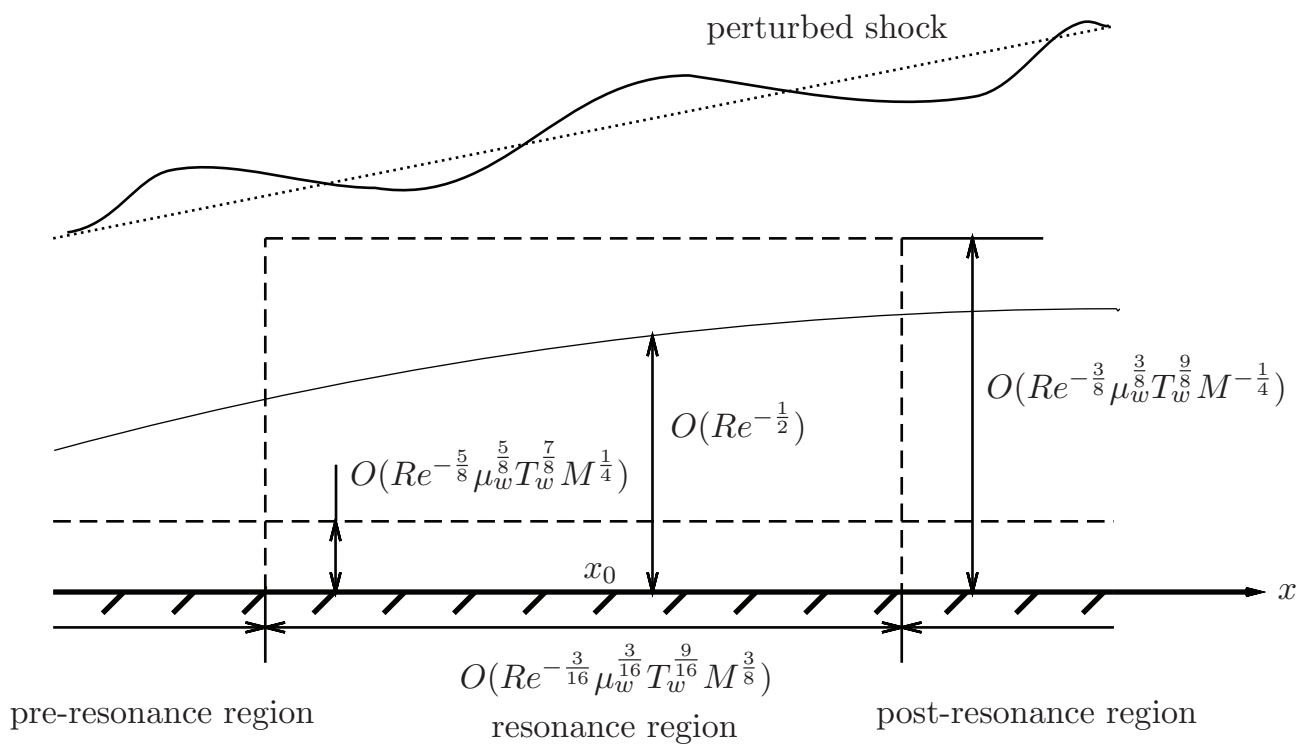

Figure 2: The asymptotic structure of the boundary-layer response and receptivity.

This relation specifies the propagation direction of the slow acoustic waves which have wavelengthes and phase speeds comparable with those of T-S waves. Consistent with the scalings (2.31), we introduce the time, the streamwise and spanwise variables

$$
\bar{t}=R e^{\frac{1}{4}} \mu_{w}^{-\frac{1}{4}} T_{w}^{-\frac{3}{4}} M^{-\frac{1}{2}} t, \quad(\bar{x}, \bar{z})=R e^{\frac{3}{8}} \mu_{w}^{-\frac{3}{8}} T_{w}^{-\frac{9}{8}} M^{-\frac{3}{4}}(x, M z) .
$$

In order for a slow acoustic wave on the scales (2.31) to arise downstream, upstream (fast or slow) acoustic, vortical and entropy modes must have appropriate scales. For all these modes, the wavenumber parallel to the shock

$$
\alpha \approx \alpha_{a}=R e^{\frac{3}{8}} \mu_{w}^{-\frac{3}{8}} T_{w}^{-\frac{9}{8}} M^{-\frac{3}{4}} \hat{\alpha}_{a}
$$

but that normal to the shock is different. For a fast/slow upstream acoustic mode, it follows from (2.13) and (2.29) that to leading order accuracy,

$$
\nu_{0}^{ \pm} \approx R^{\frac{3}{8}} \mu_{w}^{-\frac{3}{8}} T_{w}^{-\frac{9}{8}} M^{-\frac{3}{4}}\left(\hat{\alpha}_{a} \pm \sqrt{\frac{2-\bar{\gamma}}{2}} \hat{\beta}_{a}\right) / \sigma .
$$

For upstream vorticity and entropy modes, we find from (2.16) that

$$
\nu_{I} \approx R e^{\frac{3}{8}} \mu_{w}^{-\frac{3}{8}} T_{w}^{-\frac{9}{8}} M^{-\frac{3}{4}} \hat{\alpha}_{a} / \sigma .
$$

At a general streamwise location $x$ where $\left(\omega_{a}, \alpha_{a}, \beta_{a}\right) \neq\left(\omega_{\mathrm{TS}}, \alpha_{\mathrm{TS}}, \beta_{\mathrm{TS}}\right)$, an acoustic wave generates a finite response within the boundary layer. However, for each frequency $\omega_{a}$, there exists a location $x_{0}$ at which a T-S wave with the same frequency and spanwise wavenumber is neutrally stable if the streamwise wavenumber of the slow acoustic wave also coincides with that of the neutral T-S mode, that is,

$$
\hat{\omega}_{a}=\hat{\omega}_{\mathrm{TS}}, \quad \hat{\alpha}_{a}=\hat{\alpha}_{\mathrm{TS}}, \quad \hat{\beta}_{a}=\hat{\beta}_{\mathrm{TS}}
$$


at $x_{0}$, the response becomes unbounded, signaling that a resonance takes place in the vicinity of $x_{0}$. A scaling argument similar to that of $\mathrm{Wu}(2001 \mathrm{~b})$ shows that the forced response evolves into a T-S mode under the influence of non-parallelism, and this resonance region corresponds to $x-x_{0}=O(\bar{\epsilon})$ with

$$
\bar{\epsilon}=R e^{-\frac{3}{16}} \mu_{w}^{\frac{3}{16}} T_{w}^{\frac{9}{16}} M^{\frac{3}{8}} .
$$

In order to describe the evolution of the disturbance through the resonance region, we introduce

$$
x_{1}=\left(x-x_{0}\right) / \bar{\epsilon} .
$$

It follows from (2.34) and (2.40) that

$$
\frac{\partial}{\partial t} \rightarrow R e^{\frac{1}{4}} \mu_{w}^{-\frac{1}{4}} T_{w}^{-\frac{3}{4}} M^{-\frac{1}{2}} \frac{\partial}{\partial \bar{t}}, \quad \frac{\partial}{\partial z} \rightarrow \bar{\epsilon}^{-2} M \frac{\partial}{\partial \bar{z}}, \quad \frac{\partial}{\partial x} \rightarrow \bar{\epsilon}^{-2}\left(\frac{\partial}{\partial \bar{x}}+\bar{\epsilon} \frac{\partial}{\partial x_{1}}\right) .
$$

We further expand $\lambda$ about $x_{0}$,

$$
\lambda=\lambda_{0}+\bar{\epsilon} \lambda_{1} x_{1} \quad \text { with } \quad \lambda_{1}=-\frac{1}{2} \chi x_{0}^{-\frac{3}{2}} .
$$

The asymptotic structure describing the boundary-layer response and receptivity is shown in figure 2. To a sound wave with its frequency and wavenumbers being scaled as (2.31), the boundary-layer response acquires, in the wall-normal direction, the triple-deck structure that governs the inherent boundary-layer instability. A disturbance satisfying the resonance condition (2.38) evolves, in the streamwise direction, through the pre-resonance, resonance and post-resonance regimes. The response in the pre-resonance regime will be considered in the next section, and the main result is the expressions (3.21) for $a_{I}$ and $a_{R}$, the magnitudes of the pressure waves entering, and reflected by, the boundary layer respectively. The evolution in the resonance region will be analysed in $\S 4$ and the main outcome is (4.35), which describes the development of the response into a T-S wave, and allows us to determine the initial amplitude of the eigen mode excited.

\section{Pre-resonance region: boundary-layer response to free- stream disturbances}

For acoustic waves with wavelength comparable with the boundary-layer thickness, the response is essentially inviscid, and can be predicted by using the Rayleigh equation. However, for acoustic waves with long wavelengthes and low frequencies as specified by (2.31), the response is viscous and is described by triple-deck theory.

\subsection{Upper deck}

In the upper deck, the transverse variable is

$$
\bar{y}=R e^{\frac{3}{8}} \mu_{w}^{-\frac{3}{8}} T_{w}^{-\frac{9}{8}} M^{\frac{1}{4}} y,
$$

and the expansion takes the form

$$
(u, v, w, p, \rho, T)=\left(1,0,0,1 / \gamma M^{2}, 1,1\right)+\delta\left(u_{a}, M v_{a}, M w_{a}, p_{a}, M^{2} \rho_{a}, M^{2} \tau_{a}\right)+\cdots .
$$


Here we assume that there is no pre-existing T-S wave. In the pre-resonance region, no T-S wave is excited yet and so the $O(\delta / \bar{\epsilon})$ term is absent. The perturbation is solely induced by the $O(\delta)$ disturbance in the oncoming flow.

We focus on the acoustic part of the perturbation. Vortical and entropy waves are also present (see Appendix A), but they are absorbed by a thin layer located at the outer edge of the boundary layer without entering the latter (Dong \& Wu 2013; see also Maslov et al. 2010, where such features were observed experimentally). The acoustic wave is governed by the (quasi-steady) Euler equations linearized about the uniform flow, among which the momentum equation in the $y$-direction reads

$$
\frac{\partial v_{a}}{\partial \bar{x}}=-\frac{\partial p_{a}}{\partial \bar{y}} .
$$

The linearized Euler equations can be reduced to a single equation for $p_{a}$,

$$
\frac{\partial^{2} p_{a}}{\partial \bar{y}^{2}}-\frac{\partial^{2} p_{a}}{\partial \bar{x}^{2}}+\frac{\partial^{2} p_{a}}{\partial \bar{z}^{2}}=0
$$

Equations (3.4) and (3.3) can be solved in sequence to find the solution for $p_{a}$ and $v_{a}$,

$$
p_{a}=\left(a_{I} \mathrm{e}^{\mathrm{i} \hat{\gamma}{ }_{a} \bar{y}}+a_{R} \mathrm{e}^{-\mathrm{i} \hat{\gamma}_{a} \bar{y}}\right) E_{a}+\text { c.c. }, \quad v_{a}=-\frac{\hat{\gamma}_{a}}{\hat{\alpha}_{a}}\left(a_{I} \mathrm{e}^{\mathrm{i} \hat{\gamma} a \bar{y}}-a_{R} \mathrm{e}^{-\mathrm{i} \hat{\gamma} a \bar{y}}\right) E_{a}+\text { c.c. },
$$

where $E_{a}=\exp \left\{\mathrm{i}\left(\hat{\alpha}_{a} \bar{x}+\hat{\beta}_{a} \bar{z}-\hat{\omega}_{a} \bar{t}\right)\right\}$, and $a_{I}$ and $a_{R}$ depend on $x$ parametrically, and they represent respectively the amplitudes of the sound waves propagating towards and away from the wall. As $\bar{y} \rightarrow 0$,

$$
p_{a} \rightarrow\left(a_{I}+a_{R}\right) E_{a}+\text { c.c. }, \quad v_{a} \rightarrow-\frac{\hat{\gamma}_{a}}{\hat{\alpha}_{a}}\left(a_{I}-a_{R}\right) E_{a}+\text { c.c. } .
$$

In the pre-resonance stage, the T-S signature is absent and thus the shock condition corresponds to (A.20) with the modulation terms being set to zero, which further reduces, for the disturbance on the triple-deck scales, to (A.43). Noting that $\hat{a}_{I}=a_{I} \mathrm{e}^{\mathrm{i} \hat{\gamma}_{a} \bar{y}_{s}}$ and $\hat{a}_{R}=a_{R} \mathrm{e}^{-\mathrm{i} \hat{\gamma}_{a} \bar{y}_{s}}$ (which are similar to $(2.27)$ ), we obtain

$$
a_{I} \mathrm{e}^{\mathrm{i} \hat{\gamma}_{a} \bar{y}_{s}}+a_{R} \mathrm{e}^{-\mathrm{i} \hat{\gamma}_{a} \bar{y}_{s}}=J_{0},
$$

where $\bar{y}_{s}=R e^{\frac{3}{8}} \mu_{w}^{-\frac{3}{8}} T_{w}^{-\frac{9}{8}} M^{\frac{1}{4}} y_{s}$ is the rescaled shock position, and $J_{0}$ is defined in (A.44).

\subsection{Main deck}

In the main deck, the expansion takes the form

$$
\begin{gathered}
u=U_{B}(\tilde{Y})+\delta \operatorname{Re}^{\frac{1}{8}} \mu_{w}^{\frac{3}{8}} T_{w}^{\frac{9}{8}} M^{\frac{7}{4}} B_{a} U_{B}^{\prime}(\tilde{Y}) E_{a}+\text { c.c. }+\cdots, \\
v=-\delta M \mathrm{i} \hat{\alpha}_{a} B_{a} U_{B}(\tilde{Y}) E_{a}+\text { c.c. }+\cdots,
\end{gathered}
$$

where $B_{a}=B_{a}(x)$ depends on $x$ parametrically. It can be shown that

$$
\begin{gathered}
u \rightarrow \lambda \mu_{w}^{-\frac{1}{2}} T_{w}^{-\frac{1}{2}} \tilde{Y}+\delta \operatorname{Re}^{\frac{1}{8}} \mu_{w}^{-\frac{1}{8}} T_{w}^{\frac{5}{8}} M^{\frac{7}{4}} B_{a} \lambda E_{a}+\text { c.c. as } \tilde{Y} \rightarrow 0, \\
v \rightarrow-\delta M \mathrm{i} \hat{\alpha}_{a} B_{a} E_{a}+\text { c.c. } \quad \text { as } \quad \tilde{Y} \rightarrow \infty .
\end{gathered}
$$

Matching with the normal velocity at the bottom of the upper deck, as given in (3.6), yields

$$
\frac{\hat{\gamma}_{a}}{\hat{\alpha}_{a}}\left(a_{I}-a_{R}\right)=\mathrm{i} \hat{\alpha}_{a} B_{a} .
$$




\subsection{Lower deck}

In the lower deck, the appropriate transverse coordinate is defined as

$$
Y=R e^{\frac{5}{8}} \mu_{w}^{-\frac{5}{8}} T_{w}^{-\frac{7}{8}} M^{-\frac{1}{4}} y
$$

and the solution can be written as

$$
\left.\begin{array}{c}
u=R e^{-\frac{1}{8}} \mu_{w}^{\frac{1}{8}} T_{w}^{\frac{3}{8}} M^{\frac{1}{4}} \lambda Y+\delta R e^{\frac{1}{8}} \mu_{w}^{-\frac{1}{8}} T_{w}^{\frac{5}{8}} M^{\frac{7}{4}} \tilde{U}_{a} E_{a}+\text { c.c. }+\cdots, \\
v=\delta R e^{-\frac{1}{8}} \mu_{w}^{\frac{1}{8}} T_{w}^{\frac{3}{8}} M^{\frac{5}{4}} \tilde{V}_{a} E_{a}+\text { c.c. }+\cdots, \\
w=\delta R e^{\frac{1}{8}} \mu_{w}^{-\frac{1}{8}} T_{w}^{\frac{5}{8}} M^{\frac{3}{4}} \tilde{W}_{a} E_{a}+\text { c.c. }+\cdots \\
p=\delta \tilde{P}_{a} E_{a}+\text { c.c. }+\cdots
\end{array}\right\}
$$

Substituting the expansion into (2.4), we obtain, at leading order, the system of equations

$$
\left.\begin{array}{c}
\mathrm{i} \hat{\alpha}_{a} \tilde{U}_{a}+\tilde{V}_{a, Y}+\mathrm{i} \hat{\beta}_{a} \tilde{W}_{a}=0, \\
\left(\hat{\alpha}_{a} \lambda Y-\hat{\omega}_{a}\right) \tilde{U}_{a}+\lambda \tilde{V}_{a}=\tilde{U}_{a, Y Y}, \\
-\hat{W}_{a}=-\mathrm{i} \hat{\beta}_{a} \tilde{P}_{a}+\tilde{W}_{a, Y Y} .
\end{array}\right\}
$$

The above system is subject to the no-slip condition on the wall and the matching requirement with the main deck: $\tilde{U}_{a}=\tilde{V}_{a}=\tilde{W}_{a}=0$ at $Y=0$ and $\left(\tilde{U}_{a}, \tilde{W}_{a}\right) \rightarrow\left(\lambda B_{a}, 0\right)$ as $Y \rightarrow \infty$, or equivalently,

$$
\tilde{V}_{a, Y Y Y}(0)=\hat{\beta}_{a}^{2} \tilde{P}_{a} ; \quad \tilde{V}_{a, Y} \rightarrow-\mathrm{i} \hat{\alpha}_{a} \lambda B_{a} \quad \text { as } \quad Y \rightarrow \infty .
$$

Eliminating the pressure yields $\left[\partial^{2} / \partial Y^{2}-\mathrm{i}\left(\hat{\alpha}_{a} \lambda Y-\hat{\omega}_{a}\right)\right] \tilde{V}_{a, Y Y}=0$, which has the solution

$$
\tilde{V}_{a, Y}=q_{a} \int_{\tilde{\eta}_{0}}^{\tilde{\eta}} \operatorname{Ai}(\eta) \mathrm{d} \eta
$$

where $q_{a}$ is an unknown function of $x$, Ai denotes the Airy function and

$$
\tilde{\eta}=\left(\mathrm{i} \hat{\alpha}_{a} \lambda\right)^{1 / 3} Y+\tilde{\eta}_{0}, \quad \tilde{\eta}_{0}=-\mathrm{i} \hat{\omega}_{a}\left(\mathrm{i} \hat{\alpha}_{a} \lambda\right)^{-2 / 3} .
$$

Application of the boundary conditions (3.16) gives

$$
\left(\mathrm{i} \hat{\alpha}_{a} \lambda\right)^{2 / 3} \operatorname{Ai}^{\prime}\left(\tilde{\eta}_{0}\right) q_{a}=\hat{\beta}_{a}^{2} \tilde{P}_{a}, \quad q_{a} \int_{\tilde{\eta}_{0}}^{\infty} \operatorname{Ai}(\eta) \mathrm{d} \eta=-\mathrm{i} \hat{\alpha}_{a} \lambda B_{a} .
$$

That the pressure does not change across the main deck implies that

$$
a_{I}+a_{R}=\tilde{P}_{a} .
$$

Equations (3.7), (3.12) and (3.19)-(3.20) are solved to give

$$
a_{I, R}\left(\hat{\omega}_{a}, \hat{\alpha}_{a}, \hat{\beta}_{a} ; x\right)=-\frac{1}{2} J_{0}\left[\lambda^{5 / 3} \hat{\gamma}_{a} \operatorname{Ai}^{\prime}\left(\tilde{\eta}_{0}\right) \pm \mathrm{i}\left(\mathrm{i} \hat{\alpha}_{a}\right)^{1 / 3} \hat{\beta}_{a}^{2} \int_{\tilde{\eta}_{0}}^{\infty} \operatorname{Ai}(\eta) \mathrm{d} \eta\right] / \Delta,
$$

where the \pm signs refer to the subscripts $I$ and $R$ respectively, and

$$
\Delta\left(\hat{\omega}_{a}, \hat{\alpha}_{a}, \hat{\beta}_{a} ; x\right)=\left(\mathrm{i} \hat{\alpha}_{a}\right)^{1 / 3} \hat{\beta}_{a}^{2} \sin \left(\hat{\gamma}_{a} \bar{y}_{s}\right) \int_{\tilde{\eta}_{0}}^{\infty} \operatorname{Ai}(\eta) \mathrm{d} \eta-\lambda^{5 / 3} \hat{\gamma}_{a} \operatorname{Ai}^{\prime}\left(\tilde{\eta}_{0}\right) \cos \left(\hat{\gamma}_{a} \bar{y}_{s}\right)
$$


is the dispersion function of the T-S wave (Cowley \& Hall 1990) as it will transpire. We introduce a reflection coefficient

$$
\mathscr{R} \equiv a_{R} / a_{I}=\frac{2 \mathrm{i} \sin \left(\hat{\gamma}_{a} \bar{y}_{s}\right) \lambda^{5 / 3} \hat{\gamma}_{a} \operatorname{Ai}^{\prime}\left(\tilde{\eta}_{0}\right)}{\left[\mathrm{i} \sin \left(\hat{\gamma}_{a} \bar{y}_{s}\right)-\cos \left(\hat{\gamma}_{a} \bar{y}_{s}\right)\right] \lambda^{5 / 3} \hat{\gamma}_{a} \operatorname{Ai}^{\prime}\left(\tilde{\eta}_{0}\right)-\Delta}-1 .
$$

We now examine the behavior of $a_{I}$ and $a_{R}$ when the resonant condition (2.38) is satisfied at $x_{0}$, where $\Delta\left(\hat{\omega}_{a}, \hat{\alpha}_{a}, \hat{\beta}_{a} ; x_{0}\right)=0$. A Taylor expansion of the expression (3.22) about $x_{0}$ shows that

$$
a_{I, R} \rightarrow\left[\frac{x_{0} J_{0}\left[\tan \left(\hat{\gamma}_{\mathrm{TS}} \bar{y}_{s}\right) \pm \mathrm{i}\right]}{b \cos \left(\hat{\gamma}_{\mathrm{TS}} \bar{y}_{s}\right)} \int_{\eta_{0}}^{\infty} \operatorname{Ai}(\eta) \mathrm{d} \eta\right]\left(x-x_{0}\right)^{-1} \text { as } x \rightarrow x_{0},
$$

where

$$
\begin{aligned}
b= & \frac{2}{3} \tan \left(\hat{\gamma}_{\mathrm{TS}} \bar{y}_{s}\right) \eta_{0} \operatorname{Ai}\left(\eta_{0}\right)-\tan \left(\hat{\gamma}_{\mathrm{TS}} \bar{y}_{s}\right) \int_{\eta_{0}}^{\infty} \operatorname{Ai}(\eta) \mathrm{d} \eta \\
& +\frac{2 \hat{\gamma}_{\mathrm{TS}}\left(\mathrm{i} \hat{\alpha}_{\mathrm{TS}} \lambda_{0}\right)^{5 / 3}}{3 \hat{\alpha}_{\mathrm{TS}}^{2} \hat{\beta}_{\mathrm{TS}}^{2}}\left[\operatorname{Ai}^{\prime}\left(\eta_{0}\right)-\eta_{0}^{2} \operatorname{Ai}\left(\eta_{0}\right)\right] .
\end{aligned}
$$

As (3.24) indicates, the amplitudes $a_{I}$ and $a_{R}$ blow up on approaching the neutral point $x_{0}$. In the $O(\bar{\epsilon})$ vicinity of the neutral point, the rate of change of the forced response, $a_{I}^{\prime} / a_{I}$ and $a_{R}^{\prime} / a_{R}$, becomes comparable with the growth rate of the T-S mode, implying that the streamwise variable no longer plays the role of a parameter but acts a variable (Wu 1999) and consequently the preceding analysis is no longer valid. Non-parallelism strongly affects the evolution of the disturbance in this resonance region, which will be considered in the next section.

\section{Resonance region: generation of T-S waves}

The resonance region corresponds to $x_{1}=O(1)$. A valid solution is now sought by taking into account non-parallelism and the T-S wave that is emerging from the forced solution.

\subsection{Upper deck}

In the upper deck, the expansion takes the form

$$
\begin{aligned}
(u, v, w, p, \rho, T)= & \left(1,0,0,1 / \gamma M^{2}, 1,1\right)+(\delta / \bar{\epsilon})\left(u_{\mathrm{TS}}, M v_{\mathrm{TS}}, M w_{\mathrm{TS}}, p_{\mathrm{TS}}, M^{2} \rho_{\mathrm{TS}}, M^{2} \tau_{\mathrm{TS}}\right) \tilde{E} \\
& +(\delta / \bar{\epsilon}) M\left(\sigma u_{v}, v_{v}, \sigma M w_{v}, 0, \sigma^{-1} \rho_{e}, \sigma^{-1} \tau_{e}\right) E_{v} \\
& +\delta\left(u_{2}, M v_{2}, M w_{2}, p_{2}, M^{2} \rho_{2}, M^{2} \tau_{2}\right)+\text { c.c. }+\cdots
\end{aligned}
$$

where the $O(\delta / \bar{\epsilon})$ terms with the subscripts 'TS' stand for the signature of the T-S mode that is generated through the resonance, and its carrier wave

$$
\tilde{E}=\exp \left\{\mathrm{i}\left(\hat{\alpha}_{\mathrm{TS}} \bar{x}+\hat{\beta}_{\mathrm{TS}} \bar{z}-\hat{\omega}_{\mathrm{TS}} \bar{t}\right)\right\} ;
$$

the terms of the same order-of-magnitude but with the subscript ' $v$ ' denote the vorticityentropy waves, which arise along with the T-S mode in order to satisfy the shock condition, and their carrier is

$$
E_{v}=\exp \left\{\mathrm{i}\left(R e^{-\frac{1}{8}} \mu_{w}^{\frac{1}{8}} T_{w}^{\frac{3}{8}} M^{\frac{1}{4}} \hat{\alpha}_{v} \bar{x}+\hat{\beta}_{\mathrm{TS}} \bar{z}-\hat{\omega}_{\mathrm{TS}} \bar{t}\right)\right\},
$$


where $\hat{\alpha}_{v}$ is the scaled streamwise wavenumber.

Substitution of (4.1) into (2.4) yields, at leading order, the linearized Euler equations. The pressure $p_{\mathrm{TS}}$ and the normal velocity $v_{\mathrm{TS}}$ satisfy

$$
\frac{\partial^{2} p_{\mathrm{TS}}}{\partial \bar{y}^{2}}+\left(\hat{\alpha}_{\mathrm{TS}}^{2}-\hat{\beta}_{\mathrm{TS}}^{2}\right) p_{\mathrm{TS}}=0, \quad \mathrm{i} \hat{\alpha}_{\mathrm{TS}} v_{\mathrm{TS}}=-p_{\mathrm{TS}, \bar{y}}
$$

The solution for $p_{\mathrm{TS}}$ is found as

$$
p_{\mathrm{TS}}=A\left(x_{1}\right)\left[\bar{a}_{I} \mathrm{e}^{\mathrm{i} \hat{\gamma}_{\mathrm{TS}} \bar{y}}+\bar{a}_{R} \mathrm{e}^{-\mathrm{i} \hat{\gamma} \mathrm{TS} \bar{y}}\right]
$$

where $\hat{\gamma}_{\mathrm{TS}}=\left(\hat{\alpha}_{\mathrm{TS}}^{2}-\hat{\beta}_{\mathrm{TS}}^{2}\right)^{1 / 2}, \bar{a}_{I}$ and $\bar{a}_{R}$ are constants to be determined, and $A\left(x_{1}\right)$ is the amplitude of the T-S wave. The form of the solution indicates that shock-induced T-S modes exhibit the character of sound in the upper deck. The shock condition (A.17) implies that $p_{\mathrm{TS}}=0$ at $\bar{y}=\bar{y}_{s}$, and thus (Cowley \& Hall 1990)

$$
\bar{a}_{I} \mathrm{e}^{\mathrm{i} \hat{\gamma}_{\mathrm{TS}} \bar{y}_{s}}+\bar{a}_{R} \mathrm{e}^{-\mathrm{i} \hat{\gamma}_{\mathrm{TS}} \bar{y}_{s}}=0,
$$

use of which in (4.3) gives

$$
p_{\mathrm{TS}}=A\left(x_{1}\right) \bar{a}_{I}\left(\mathrm{e}^{\mathrm{i} \hat{\gamma}_{\mathrm{TS}} \bar{y}}-\mathrm{e}^{2 \mathrm{i} \hat{\gamma}_{\mathrm{TS}} \bar{y}_{s}-\mathrm{i} \hat{\gamma}_{\mathrm{TS}} \bar{y}}\right) .
$$

It follows from the transverse momentum equation in (4.2) that

$$
v_{\mathrm{TS}}=-\frac{\hat{\gamma}_{\mathrm{TS}}}{\hat{\alpha}_{\mathrm{TS}}} \bar{a}_{I} A\left(x_{1}\right)\left(\mathrm{e}^{\mathrm{i} \hat{\gamma}_{\mathrm{TS}} \bar{y}}+\mathrm{e}^{2 \mathrm{i} \hat{\gamma}_{\mathrm{TS}} \bar{y}_{s}-\mathrm{i} \hat{\gamma}_{\mathrm{TS}} \bar{y}}\right) .
$$

Now turn to the vorticity-entropy waves, which accompany the T-S mode. Comparing (4.1) with (2.17)-(2.18) and noting (A.4) and (A.18), we find that

$$
\left(u_{v}, v_{v}, w_{v}, \rho_{e}, \tau_{e}\right)=2 \bar{a}_{I} A\left(x_{1}\right)\left(\hat{\alpha}_{\mathrm{TS}},-\hat{\beta}_{\mathrm{TS}}^{2} / \hat{\alpha}_{\mathrm{TS}}, \hat{\beta}_{\mathrm{TS}}, \hat{\alpha}_{\mathrm{TS}},-\hat{\alpha}_{\mathrm{TS}}\right) \mathrm{e}^{-\mathrm{i} \hat{\gamma}_{v} \bar{y}} / \sqrt{\hat{\alpha}_{\mathrm{TS}}^{2}-\hat{\beta}_{\mathrm{TS}}^{2}} .
$$

By rewriting (A.4) in terms of $\bar{x}$ and $\bar{y}$, and using the dispersion relation (A.5), the vertical wavenumber, $\hat{\gamma}_{v} \equiv-R e^{-\frac{3}{8}} \mu_{w}^{\frac{3}{8}} T_{w}^{\frac{9}{8}} M^{-\frac{1}{4}}\left[\alpha \sin \phi+\nu_{s} \cos \phi\right]$, is found to be

$$
\hat{\gamma}_{v} \approx\left[\hat{\alpha}-R e^{-\frac{1}{8}} \mu_{w}^{\frac{1}{8}} T_{w}^{\frac{3}{8}} M^{\frac{1}{4}} \hat{\omega}_{\mathrm{TS}}-\bar{\epsilon} \mathrm{i} A^{\prime}\left(x_{1}\right) / A\right] /(M V) \approx-(\sigma M) \hat{\alpha}_{\mathrm{TS}},
$$

while the streamwise wavenumber

$$
\hat{\alpha}_{v}=\hat{\omega}_{\mathrm{TS}}+R e^{-\frac{1}{16}} \mu_{w}^{\frac{1}{16}} T_{w}^{\frac{3}{16}} M^{\frac{1}{8}} \mathrm{i} A^{\prime} / A,
$$

consistent with the advective equations satisfied by vorticity-entropy waves in the system $(x, y, z)$. It is worth noting that the streamwise and spanwise velocity components of the vorticity wave are much greater than those of the T-S wave. As its phase speed is approximately unity, this part of disturbance is trapped in a thin layer at the outer reach of the boundary layer and hence has little influence on the latter (Dong \& Wu 2013).

For the $O(\delta)$ disturbance in the expansion (4.1), it suffices to consider $p_{2}$ and $v_{2}$ only, which are governed by the equations

$$
\frac{\partial^{2} p_{2}}{\partial \bar{y}^{2}}-\frac{\partial^{2} p_{2}}{\partial \bar{x}^{2}}+\frac{\partial^{2} p_{2}}{\partial \bar{z}^{2}}=2 \mathrm{i} \hat{\alpha}_{\mathrm{TS}} \bar{a}_{I} A^{\prime}\left(x_{1}\right)\left(\mathrm{e}^{\mathrm{i} \hat{\gamma}_{\mathrm{TS}} \bar{y}}-\mathrm{e}^{2 \mathrm{i} \hat{\gamma}_{\mathrm{TS}} \bar{y}_{s}-\mathrm{i} \hat{\gamma}_{\mathrm{TS}} \bar{y}}\right) \tilde{E},
$$




$$
\frac{\partial v_{2}}{\partial \bar{x}}=-\frac{\partial p_{2}}{\partial \bar{y}}-\frac{\partial v_{\mathrm{TS}}}{\partial x_{1}} \tilde{E}
$$

The solution for $p_{2}$ and $v_{2}$ takes the form $\left(p_{2}, v_{2}\right)=\left(\hat{P}_{2}, \hat{V}_{2}\right) \tilde{E}+$ c.c., where $\hat{P}_{2}$ and $\hat{V}_{2}$ are found from (4.9) and (4.10) as

$$
\begin{gathered}
\hat{P}_{2}=H_{1}\left(x_{1}\right) \mathrm{e}^{\mathrm{i} \hat{\gamma}_{\mathrm{TS}} \bar{y}}+H_{2}\left(x_{1}\right) \mathrm{e}^{-\mathrm{i} \hat{\gamma}_{\mathrm{TS}} \bar{y}}+\frac{\hat{\alpha}_{\mathrm{TS}}}{\hat{\gamma}_{\mathrm{TS}}} \bar{a}_{I} A^{\prime}\left(x_{1}\right)\left(\mathrm{e}^{\mathrm{i} \hat{\gamma}_{\mathrm{TS}} \bar{y}}+\mathrm{e}^{2 \mathrm{i} \hat{\gamma}_{\mathrm{TS}} \bar{y}_{s}-\mathrm{i} \hat{\gamma}_{\mathrm{TS}} \bar{y}}\right)\left(\bar{y}-\bar{y}_{s}\right), \\
\hat{V}_{2}=-\frac{\hat{\gamma}_{\mathrm{TS}}}{\hat{\alpha}_{\mathrm{TS}}}\left[H_{1} \mathrm{e}^{\mathrm{i} \hat{\gamma}_{\mathrm{TS}} \bar{y}}-H_{2} \mathrm{e}^{-\mathrm{i} \hat{\gamma}_{\mathrm{TS}} \bar{y}}\right]+\frac{\mathrm{i} \hat{\beta}_{\mathrm{TS}}^{2}}{\hat{\gamma}_{\mathrm{TS}} \hat{\alpha}_{\mathrm{TS}}^{2}} \bar{a}_{I} A^{\prime}\left(x_{1}\right)\left(\mathrm{e}^{\mathrm{i} \hat{\gamma}_{\mathrm{TS}} \bar{y}}+\mathrm{e}^{2 \mathrm{i} \hat{\gamma}_{\mathrm{TS}} \bar{y}_{s}-\mathrm{i} \hat{\gamma}_{\mathrm{TS}} \bar{y}}\right) \\
\quad-\bar{a}_{I} A^{\prime}\left(x_{1}\right)\left(\mathrm{e}^{\mathrm{i} \hat{\gamma}_{\mathrm{TS}} \bar{y}}-\mathrm{e}^{2 \mathrm{i} \hat{\gamma}_{\mathrm{TS}} \bar{y}_{s}-\mathrm{i} \hat{\gamma}_{\mathrm{TS}} \bar{y}}\right)\left(\bar{y}-\bar{y}_{s}\right) .
\end{gathered}
$$

Clearly, as $\bar{y} \rightarrow 0$,

$$
\begin{gathered}
p_{2} \rightarrow\left\{H_{1}+H_{2}-\frac{\hat{\alpha}_{\mathrm{TS}}}{\hat{\gamma}_{\mathrm{TS}}} \bar{a}_{I} A^{\prime}\left(1+\mathrm{e}^{2 \mathrm{i} \hat{\gamma}_{\mathrm{TS}} \bar{y}_{s}}\right) \bar{y}_{s}\right\} \tilde{E}, \\
v_{2} \rightarrow-\left\{\frac{\hat{\gamma}_{\mathrm{TS}}}{\hat{\alpha}_{\mathrm{TS}}}\left(H_{1}-H_{2}\right)-\frac{\mathrm{i} \hat{\beta}_{\mathrm{TS}}^{2}}{\hat{\gamma}_{\mathrm{TS}} \hat{\alpha}_{\mathrm{TS}}^{2}} \bar{a}_{I} A^{\prime}\left(1+\mathrm{e}^{2 \mathrm{i} \hat{\gamma}_{\mathrm{TS}} \bar{y}_{s}}\right)-\bar{a}_{I} A^{\prime}\left(1-\mathrm{e}^{2 \mathrm{i} \hat{\gamma}_{\mathrm{TS}} \bar{y}_{s}}\right) \bar{y}_{s}\right\} \tilde{E} .
\end{gathered}
$$

In addition, the shock condition (A.43) gives

$$
H_{1}\left(x_{1}\right) \mathrm{e}^{\mathrm{i} \hat{\gamma}_{\mathrm{TS}} \bar{y}_{s}}+H_{2}\left(x_{1}\right) \mathrm{e}^{-\mathrm{i} \hat{\gamma}_{\mathrm{TS}} \bar{y}_{s}}=J_{0},
$$

where $J_{0}$ is defined in (A.44) for fast/slow acoustic, vortical and entropy modes.

\subsection{Main deck}

Since no further interaction takes place in the main deck, the perturbation on the tripledeck scales arises merely as the response to the upper deck and satisfies the standard main-deck equations. The solution for the velocities is found as

$$
\begin{aligned}
& u=U_{B}(\tilde{Y})+\delta R e^{\frac{5}{16}} \mu_{w}^{\frac{3}{16}} T_{w}^{\frac{9}{16}} M^{\frac{11}{8}} B\left(x_{1}\right) U_{B}^{\prime}(\tilde{Y}) \tilde{E}+\text { c.c. }, \\
& v=-\delta R e^{-\frac{3}{16}} \mu_{w}^{\frac{3}{16}} T_{w}^{\frac{9}{16}} M^{\frac{11}{8}} \frac{\partial}{\partial x}\left\{B\left(x_{1}\right) U_{B}(\tilde{Y}) \tilde{E}+\text { c.c. }\right\} .
\end{aligned}
$$

In order to match with the upper-deck solution, the displacement function $B$ expands as $B=A\left(x_{1}\right) B_{1}+\bar{\epsilon} B_{2}\left(x_{1}\right)$ with $B_{1}$ being an undetermined constant and $B_{2}$ a function of $x_{1}$. It can be shown that

$$
\begin{gathered}
u \rightarrow \lambda \mu_{w}^{-\frac{1}{2}} T_{w}^{-\frac{1}{2}} \tilde{Y}+\delta e^{\frac{5}{16}} \mu_{w}^{-\frac{5}{16}} T_{w}^{\frac{1}{16}} M^{\frac{11}{8}}\left[\lambda_{0} A B_{1}+\bar{\epsilon}\left(\lambda_{0} B_{2}+\lambda_{1} x_{1} A B_{1}\right)\right] \tilde{E}+\text { c.c. as } \tilde{Y} \rightarrow 0, \\
v \rightarrow-\delta \operatorname{Re}^{\frac{3}{16}} \mu_{w}^{-\frac{3}{16}} T_{w}^{-\frac{9}{16}} M^{\frac{5}{8}}\left[\mathrm{i} \hat{\alpha}_{\mathrm{TS}} A B_{1}+\bar{\epsilon}\left(\mathrm{i} \hat{\alpha}_{\mathrm{TS}} B_{2}+A^{\prime} B_{1}\right)\right] \tilde{E}+\text { c.c. as } \tilde{Y} \rightarrow \infty . \quad(4.17)
\end{gathered}
$$

Matching between the upper-deck and main-deck solutions for $v$ implies that

$$
\begin{gathered}
\frac{\hat{\gamma}_{\mathrm{TS}}}{\hat{\alpha}_{\mathrm{TS}}} \bar{a}_{I}\left(1+\mathrm{e}^{2 \mathrm{i} \hat{\gamma}_{\mathrm{TS}} \bar{y}_{s}}\right)=\mathrm{i} \hat{\alpha}_{\mathrm{TS}} B_{1} \\
\frac{\hat{\gamma}_{\mathrm{TS}}}{\hat{\alpha}_{\mathrm{TS}}}\left(H_{1}-H_{2}\right)-\frac{\mathrm{i} \hat{\beta}_{\mathrm{TS}}^{2}}{\hat{\gamma}_{\mathrm{TS}} \hat{\alpha}_{\mathrm{TS}}^{2}} \bar{a}_{I} A^{\prime}\left(1+\mathrm{e}^{2 \mathrm{i} \hat{\gamma}_{\mathrm{TS}} \bar{y}_{s}}\right)-\bar{a}_{I} A^{\prime}\left(1-\mathrm{e}^{2 \mathrm{i} \hat{\gamma}_{\mathrm{TS}} \bar{y}_{s}}\right) \bar{y}_{s}=\mathrm{i} \hat{\alpha}_{\mathrm{TS}} B_{2}+A^{\prime} B_{1}
\end{gathered}
$$




\subsection{Lower deck}

In the lower deck, the solution can be written as

$$
\begin{aligned}
u= & R e^{-\frac{1}{8}} \mu_{w}^{\frac{1}{8}} T_{w}^{\frac{3}{8}} M^{\frac{1}{4}}\left(\lambda_{0}+\bar{\epsilon} \lambda_{1} x_{1}\right) Y \\
& +\delta e^{\frac{1}{8}} \mu_{w}^{-\frac{1}{8}} T_{w}^{\frac{5}{8}} M^{\frac{7}{4}} / \bar{\epsilon}\left[A\left(x_{1}\right) \tilde{U}_{1}+\bar{\epsilon} \tilde{U}_{2}\right] \tilde{E}+\text { c.c. }+\cdots, \\
v= & \delta \operatorname{Re}^{-\frac{1}{8}} \mu_{w}^{\frac{1}{8}} T_{w}^{\frac{3}{8}} M^{\frac{5}{4}} / \bar{\epsilon}\left[A\left(x_{1}\right) \tilde{V}_{1}+\bar{\epsilon} \tilde{V}_{2}\right] \tilde{E}+\text { c.c. }+\cdots \\
w= & \delta \operatorname{Re}^{\frac{1}{8}} \mu_{w}^{-\frac{1}{8}} T_{w}^{\frac{5}{8}} M^{\frac{3}{4}} / \bar{\epsilon}\left[A\left(x_{1}\right) \tilde{W}_{1}+\bar{\epsilon} \tilde{W}_{2}\right] \tilde{E}+\text { c.c. }+\cdots \\
p= & \delta / \bar{\epsilon}\left[A\left(x_{1}\right) \tilde{P}_{1}+\bar{\epsilon} \tilde{P}_{2}\right] \tilde{E}+\text { c.c. }+\cdots
\end{aligned}
$$

Substituting the expansion into (2.4), we arrive at equations

$$
\left.\begin{array}{c}
\mathrm{i} \hat{\alpha}_{\mathrm{TS}} \tilde{U}_{1}+\tilde{V}_{1, Y}+\mathrm{i} \hat{\beta}_{\mathrm{TS}} \tilde{W}_{1}=0, \\
\left.\lambda_{0} Y-\hat{\omega}_{\mathrm{TS}}\right) \tilde{U}_{1}+\lambda_{0} \tilde{V}_{1}=\tilde{U}_{1, Y Y}, \\
\left.-\hat{\omega}_{\mathrm{TS}}\right) \tilde{W}_{1}=-\mathrm{i} \hat{\beta}_{\mathrm{TS}} \tilde{P}_{1}+\tilde{W}_{1, Y Y} .
\end{array}\right\}
$$

The above system is subject to the no-slip condition on the wall, $\tilde{U}_{1}=\tilde{V}_{1}=\tilde{W}_{1}=0$ at $Y=0$, and the matching condition with the main deck, $\left(\tilde{U}_{1}, \tilde{W}_{1}\right) \rightarrow\left(\lambda_{0} B_{1}, 0\right)$ as $Y \rightarrow \infty$, which are equivalent to

$$
\tilde{V}_{1, Y Y Y}(0)=\hat{\beta}_{\mathrm{TS}}^{2} \tilde{P}_{1} ; \quad \tilde{V}_{1, Y} \rightarrow-\mathrm{i} \hat{\alpha}_{\mathrm{TS}} \lambda_{0} B_{1} \quad \text { as } \quad Y \rightarrow \infty .
$$

As in the previous section, it is found that

$$
\tilde{V}_{1, Y}=\int_{\eta_{0}}^{\eta} \operatorname{Ai}(\eta) \mathrm{d} \eta
$$

where Ai denotes the Airy function and

$$
\eta=\left(\mathrm{i} \hat{\alpha}_{\mathrm{TS}} \lambda_{0}\right)^{1 / 3} Y+\eta_{0}, \quad \eta_{0}=-\mathrm{i} \hat{\omega}_{\mathrm{TS}}\left(\mathrm{i} \hat{\alpha}_{\mathrm{TS}} \lambda_{0}\right)^{-2 / 3} .
$$

Application of the boundary conditions (4.22) gives

$$
\left(\mathrm{i} \hat{\alpha}_{\mathrm{TS}} \lambda_{0}\right)^{2 / 3} \operatorname{Ai}^{\prime}\left(\eta_{0}\right)=\hat{\beta}_{\mathrm{TS}}^{2} \tilde{P}_{1}, \quad \int_{\eta_{0}}^{\infty} \operatorname{Ai}(\eta) \mathrm{d} \eta=-\mathrm{i} \hat{\alpha}_{\mathrm{TS}} \lambda_{0} B_{1} .
$$

The fact that the pressure does not vary across the main and lower decks leads to

$$
\bar{a}_{I}\left(1-\mathrm{e}^{2 \mathrm{i} \hat{\gamma}_{\mathrm{TS}} \bar{y}_{s}}\right)=\tilde{P}_{1} .
$$

From (4.18), (4.25) and (4.26) follows the dispersion relation of T-S modes (Cowley \& Hall 1990)

$$
\Delta\left(\hat{\omega}_{\mathrm{TS}}, \hat{\alpha}_{\mathrm{TS}}, \hat{\beta}_{\mathrm{TS}} ; x_{0}\right)=0
$$

where the dispersion function $\Delta$ is defined by (3.22). The neutral wavenumbers and frequency can be determined by

$$
\hat{\alpha}_{\mathrm{TS}}=\lambda_{0}{ }^{5 / 4} \alpha_{N}, \quad \hat{\beta}_{\mathrm{TS}}=\lambda_{0}{ }^{5 / 4} \beta_{N}, \quad \hat{\omega}_{\mathrm{TS}}=\lambda_{0}{ }^{3 / 2} \omega_{N}, \quad \bar{y}_{s}=\lambda_{0}{ }^{-5 / 4} y_{N},
$$



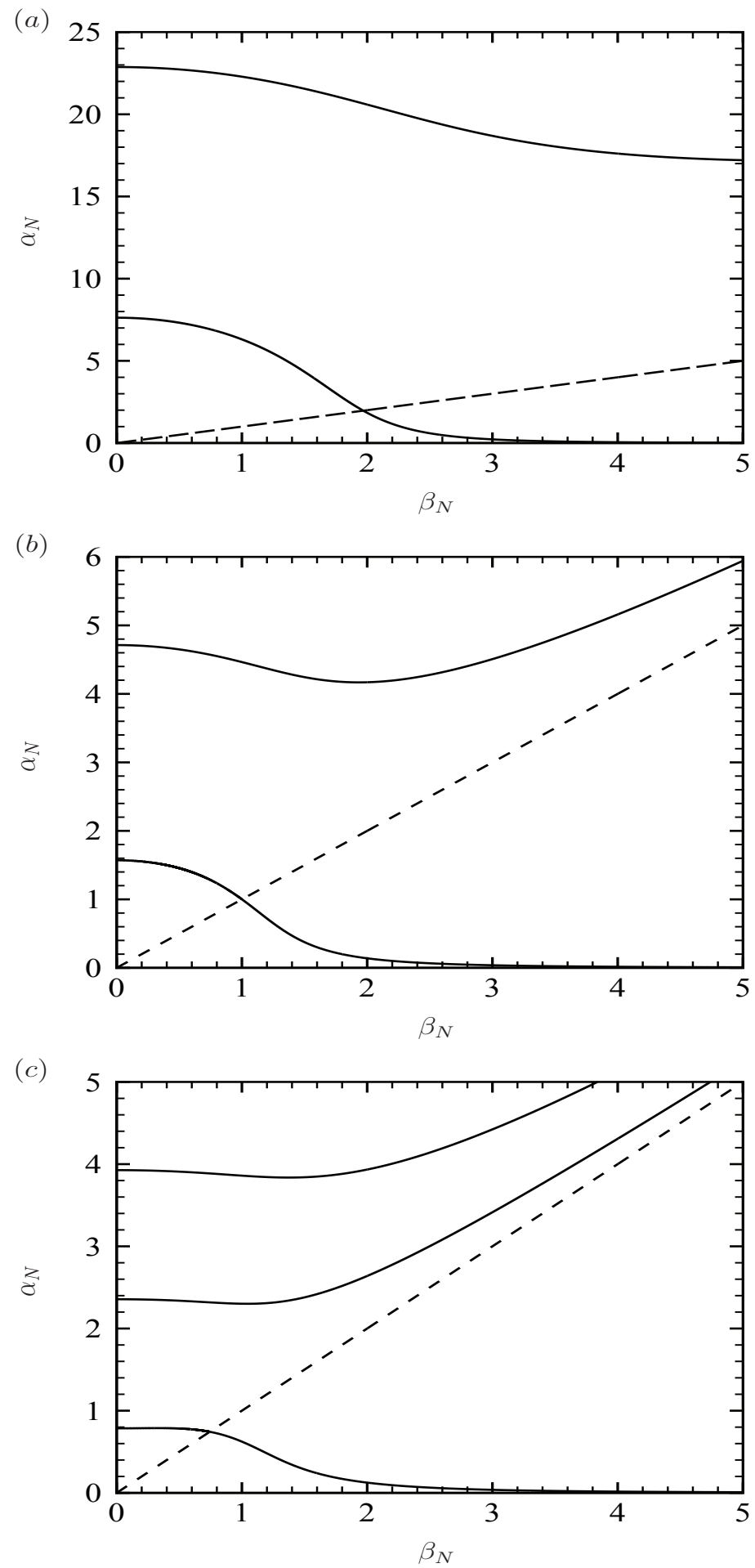

Figure 3: The neutral curves $\alpha_{N}=\alpha_{N}\left(\beta_{N}\right)$ for $(a) y_{N}=0.2,(b) y_{N}=1$ and $(c) y_{N}=2$. 
(a)

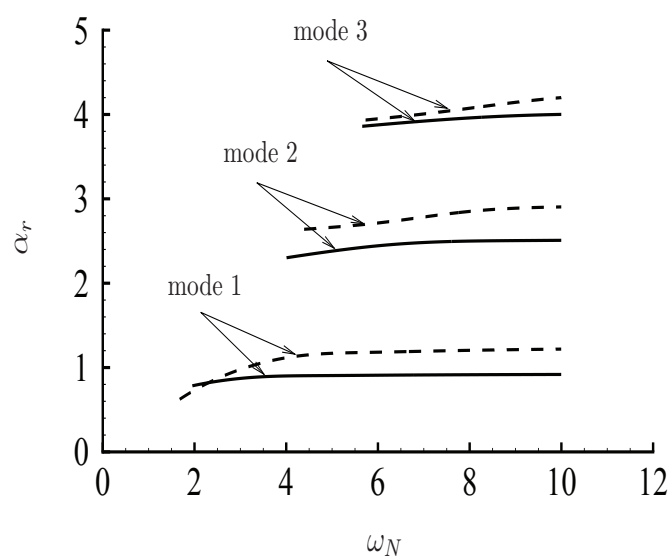

(b)

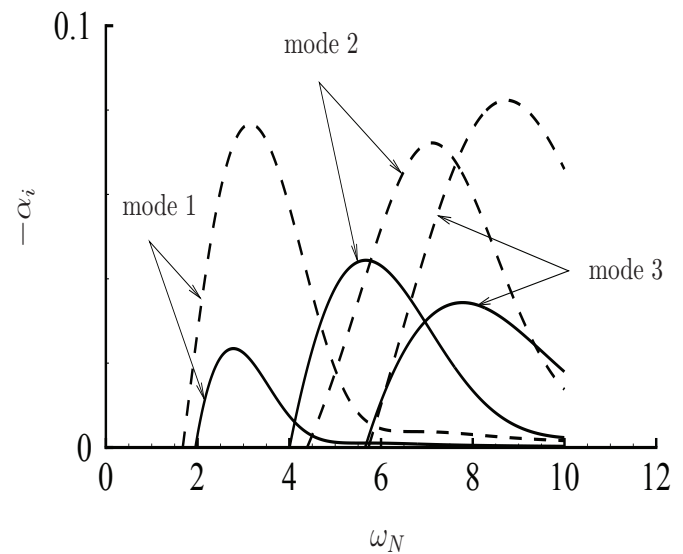

Figure 4: Normalised wavenumeber $\alpha_{r}(a)$ and normalised spatial spatial growth rate $-\alpha_{i}$ (b) versus $\omega_{N}$ calculated for $y_{N}=2$. Mode 1: solid line for $\beta_{N}=0.5$ and dashed line for $\beta_{N}=1$. Modes 2 and 3: solid lines for $\beta=1$ and dashed lines for $\beta_{N}=2$.

with $\alpha_{N}, \beta_{N}$ and $\omega_{N}$ satisfying

$$
\alpha_{N}^{1 / 3} \beta_{N}^{2} \tan \left[\left(\alpha_{N}^{2}-\beta_{N}^{2}\right)^{1 / 2} y_{N}\right]=d_{1}\left(\alpha_{N}^{2}-\beta_{N}^{2}\right)^{1 / 2}, \quad \omega_{N}=d_{2} \alpha_{N}^{2 / 3}
$$

where $d_{1} \approx 1.001$ and $d_{2} \approx 2.299$.

We computed neutral modes for several values of $y_{N}$. As is shown in figure 3 , the presence of a shock allows for a countable set of neutral modes. We denote the mode with the smallest $\alpha_{N}$ for a given $\beta_{N}$ as mode 1, and modes 2, 3 and etc. have increasingly larger $\alpha_{N}$. The bandwidth of these shock-induced instability modes appears to be inversely proportional to $y_{N}$. Their existence is restricted in the region $\beta_{N}<\alpha_{N}$ except mode-1 family, which continues to the region $\beta_{N}>\alpha_{N}$. Modes in this region approach, in the limit $y_{N} \rightarrow \infty$, the more familiar T-S modes whose eigenfunctions attenuate in the upper deck (Smith 1989).

Cowley \& Hall (1990) calculated only temporal growth rates. Here we perform a spatial stability analysis, which is considered to be more relevant. Figure 4 displays the wavenumers and spatial amplification rates of modes 1,2 and 3 . For a fixed $\beta_{N}$, as $\omega_{N}$ is increased from the neutral frequency, the wavenumber increases; the growth rate increases first, reaches a peak and then decreases, but always remains positive. This is in contrast with the temporal growth rate, which changes its sign repeatedly as the wavenumber increases (Cowley \& Hall 1990).

In order to provide further information about the instability, we compute the growth rate $\left(-\alpha_{i}\right)$ and the associated wavenumber for modes 1 and 2 in a fairly large range of $\omega_{N}$ and $\beta_{N}$. Figures 5 and 6 show the contours of $-\alpha_{i}\left(\omega_{N}, \beta_{N}\right)$ and $\alpha_{r}\left(\omega_{N}, \beta_{N}\right)$ on the $\left(\omega_{N}, \beta_{N}\right)$ plane. Overall, the second (and higher) modes have smaller growth rates than those in mode-1 family. For each $\omega_{N}$, the most unstable mode is oblique. The shockinduced neutral modes form the lower branch of the neutral curve, the unstable region above which appears to extend to infinity suggesting that an upper branch is absent in the triple-deck scaling regime. The results imply that once a lower-branch mode is excited, it will amplify since for a mode with a fixed physical frequency and spanwise wavelength, 
(a)

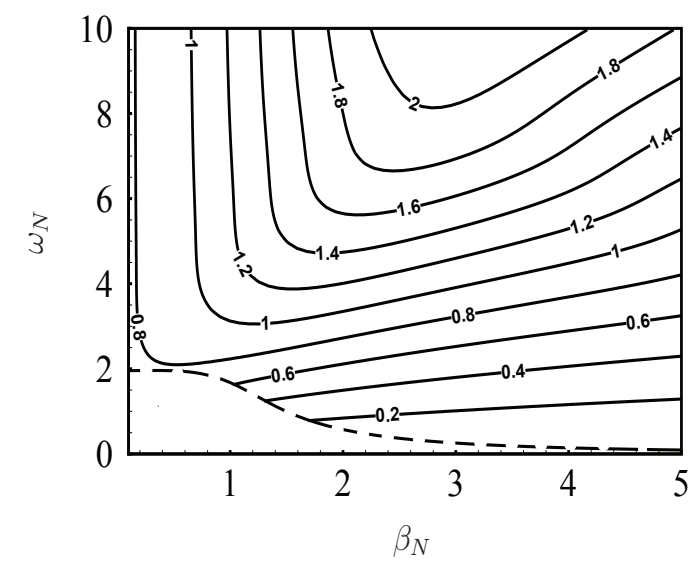

(b)

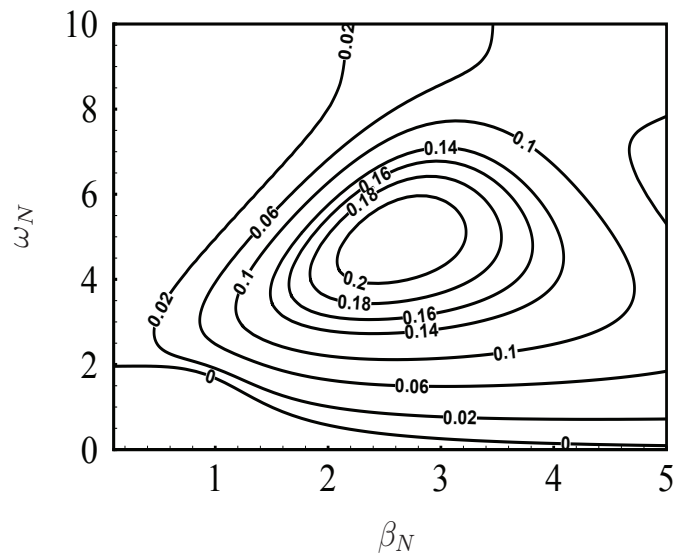

Figure 5: Contours of wavenumbers $(a)$ and spatial amplification rates $(b)$ of growing modes 1 for the case of $y_{N}=2$. The dashed line in $(a)$ marks the neutral curve, and the decaying modes below it are not considered.

(a)

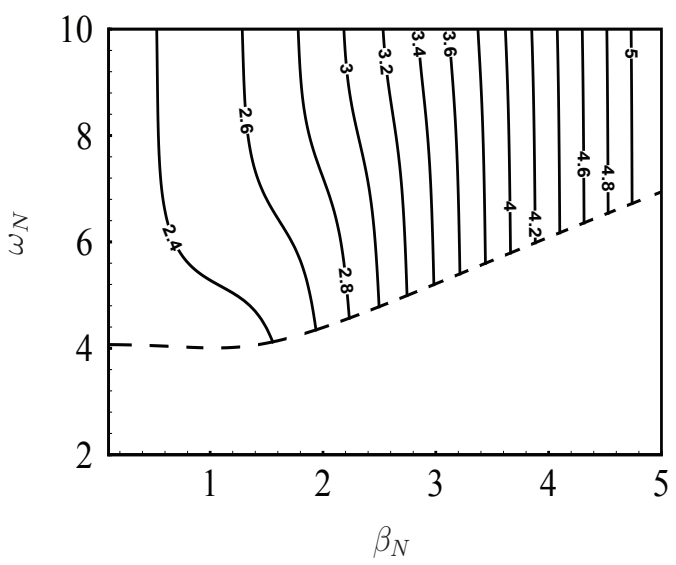

(b)

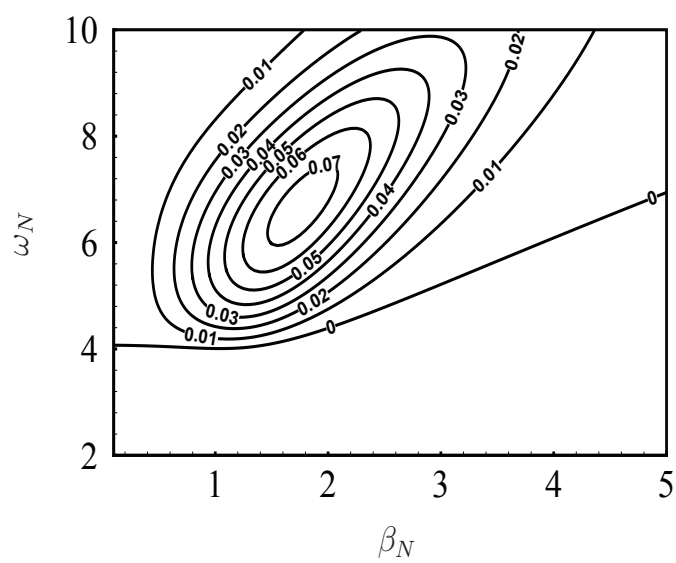

Figure 6: Contours of wavenumbers $(a)$ and spatial amplification rates $(b)$ of growing modes 2 for the case of $y_{N}=2$. The dashed line in $(a)$ marks the neutral curve, and the decaying modes below it are not considered. 
its non-dimensional frequency and spanwise wavenumber increase as the boundary layer becomes thicker.

The second-order terms in the perturbation expansion (4.20) are governed by equations

$$
\begin{aligned}
\mathrm{i} \hat{\alpha}_{\mathrm{TS}} \tilde{U}_{2}+\tilde{V}_{2, Y}+\mathrm{i} \hat{\beta}_{\mathrm{TS}} \tilde{W}_{2}= & -A^{\prime}\left(x_{1}\right) \tilde{U}_{1} \\
\mathrm{i}\left(\hat{\alpha}_{\mathrm{TS}} \lambda_{0} Y-\hat{\omega}_{\mathrm{TS}}\right) \tilde{U}_{2}+\lambda_{0} \tilde{V}_{2} & =\tilde{U}_{2, Y Y}-\Lambda\left(x_{1}\right) Y \tilde{U}_{1}-\lambda_{1} x_{1} A\left(x_{1}\right) \tilde{V}_{1}, \\
\mathrm{i}\left(\hat{\alpha}_{\mathrm{TS}} \lambda_{0} Y-\hat{\omega}_{\mathrm{TS}}\right) \tilde{W}_{2} & =-\mathrm{i} \hat{\beta}_{\mathrm{TS}} \tilde{P}_{2}+\tilde{W}_{2, Y Y}-\Lambda\left(x_{1}\right) Y \tilde{W}_{1},
\end{aligned}
$$

where $\Lambda$ is defined by

$$
\Lambda=\lambda_{0} A^{\prime}\left(x_{1}\right)+\mathrm{i} \hat{\alpha}_{\mathrm{TS}} \lambda_{1} x_{1} A\left(x_{1}\right) .
$$

The no-slip condition on the wall and matching with the main-deck solution imply that $\tilde{U}_{2}=\tilde{V}_{2}=\tilde{W}_{2}=0$ at $Y=0$ and $\left(\tilde{U}_{2}, \tilde{W}_{2}\right) \rightarrow\left(\lambda_{0} B_{2}+\lambda_{1} x_{1} A B_{1}, 0\right)$ as $Y \rightarrow \infty$, or equivalently,

$$
\tilde{V}_{2, Y Y Y}(0)=\hat{\beta}_{\mathrm{TS}}^{2} \tilde{P}_{2} ; \quad \tilde{V}_{2, Y} \rightarrow-\mathrm{i} \hat{\alpha}_{\mathrm{TS}} \lambda_{0} B_{2}-\Lambda\left(x_{1}\right) B_{1} \quad \text { as } \quad Y \rightarrow \infty .
$$

Eliminating the pressure $\tilde{P}_{2}$ from (4.30) yields

$$
\left\{\frac{\partial^{2}}{\partial Y^{2}}-\mathrm{i}\left(\hat{\alpha}_{\mathrm{TS}} \lambda_{0} Y-\hat{\omega}_{\mathrm{TS}}\right)\right\} \tilde{V}_{2, Y Y}=\Lambda\left(x_{1}\right) Y \tilde{V}_{1, Y Y}=\Lambda\left(x_{1}\right)\left(\eta-\eta_{0}\right) \operatorname{Ai}(\eta),
$$

which has the solution (cf. Wu 1999)

$$
\tilde{V}_{2, Y}=\frac{1}{3}\left(\mathrm{i} \hat{\alpha}_{\mathrm{TS}} \lambda_{0}\right)^{-1} \Lambda\left(x_{1}\right)\left[\left(\eta-3 \eta_{0}\right) \operatorname{Ai}(\eta)+2 \eta_{0} \operatorname{Ai}\left(\eta_{0}\right)\right]+q_{2} \int_{\eta_{0}}^{\eta} \operatorname{Ai}(\eta) \mathrm{d} \eta,
$$

where $q_{2}$ is an unknown function of $x_{1}$. The boundary conditions (4.31) lead to

$$
\begin{gathered}
\frac{2}{3}\left(\mathrm{i} \hat{\alpha}_{\mathrm{TS}} \lambda_{0}\right)^{-1 / 3} \Lambda\left[\mathrm{Ai}^{\prime}\left(\eta_{0}\right)-\eta_{0} \mathrm{Ai}^{\prime \prime}\left(\eta_{0}\right)\right]+\left(\mathrm{i} \hat{\alpha}_{\mathrm{TS}} \lambda_{0}\right)^{2 / 3} q_{2} \mathrm{Ai}^{\prime}\left(\eta_{0}\right)=\hat{\beta}_{\mathrm{TS}}^{2} \tilde{P}_{2}, \\
\frac{2}{3}\left(\mathrm{i} \hat{\alpha}_{\mathrm{TS}} \lambda_{0}\right)^{-1} \Lambda \eta_{0} \operatorname{Ai}\left(\eta_{0}\right)+q_{2} \int_{\eta_{0}}^{\infty} \operatorname{Ai}(\eta) \mathrm{d} \eta=-\mathrm{i} \hat{\alpha}_{\mathrm{TS}} \lambda_{0} B_{2}-\Lambda B_{1} .
\end{gathered}
$$

Since the pressure does not change across the main and lower decks, we have

$$
H_{1}+H_{2}-\frac{\hat{\alpha}_{\mathrm{TS}}}{\hat{\gamma}_{\mathrm{TS}}} \bar{a}_{I} A^{\prime}\left(1+\mathrm{e}^{2 \mathrm{i} \hat{\gamma}_{\mathrm{TS}} \bar{y}_{s}}\right) \bar{y}_{s}=\tilde{P}_{2}
$$

Eliminating $B_{2}$ and $\tilde{P}_{2}$ from (4.13), (4.19), (4.32)-(4.34) and making use of (4.25)-(4.26), we finally obtain the amplitude equation for $A$,

$$
A^{\prime}\left(x_{1}\right)=\varsigma x_{1} A+N
$$

where

$$
\varsigma=-\mathrm{i} \hat{\alpha}_{\mathrm{TS}} \lambda_{1} b /\left(\lambda_{0} a\right), \quad N=-\lambda_{0} \hat{\gamma}_{\mathrm{TS}} \sec \left(\hat{\gamma}_{\mathrm{TS}} \bar{y}_{s}\right) J_{0} / a,
$$

with constants $b$ being given by (3.25), and

$$
\begin{gathered}
a=\frac{2}{3} \tan \left(\hat{\gamma}_{\mathrm{TS}} \bar{y}_{s}\right) \eta_{0} \operatorname{Ai}\left(\eta_{0}\right)+\left[\frac{\hat{\alpha}_{\mathrm{TS}}^{2} \bar{y}_{s}}{\hat{\gamma}_{\mathrm{TS}} \cos ^{2}\left(\hat{\gamma}_{\mathrm{TS}} \bar{y}_{s}\right)}-\frac{\hat{\beta}_{\mathrm{TS}}^{2}}{\hat{\gamma}_{\mathrm{TS}}^{2}} \tan \left(\hat{\gamma}_{\mathrm{TS}} \bar{y}_{s}\right)\right] \int_{\eta_{0}}^{\infty} \operatorname{Ai}(\eta) \mathrm{d} \eta \\
+\frac{2 \hat{\gamma}_{\mathrm{TS}}\left(\mathrm{i} \hat{\alpha}_{\mathrm{TS}} \lambda_{0}\right)^{5 / 3}}{3 \hat{\alpha}_{\mathrm{TS}}^{2} \hat{\beta}_{\mathrm{TS}}^{2}}\left[\operatorname{Ai}^{\prime}\left(\eta_{0}\right)-\eta_{0}^{2} \operatorname{Ai}\left(\eta_{0}\right)\right] .
\end{gathered}
$$




\section{$5 \quad$ Matching and coupling coefficients}

\subsection{Matching with the pre-resonance region}

On assuming that the amplitude of the T-S wave vanishes as $x_{1} \rightarrow-\infty$, the amplitude equation (4.35) has the solution

$$
A\left(x_{1}\right)=N \mathrm{e}^{\varsigma x_{1}{ }^{2} / 2} \int_{-\infty}^{x_{1}} \mathrm{e}^{-\varsigma \xi^{2} / 2} \mathrm{~d} \xi .
$$

More precisely, it can be shown that

$$
A\left(x_{1}\right) \rightarrow(N / \varsigma)\left(-x_{1}\right)^{-1}+O\left(x_{1}^{-3}\right) \text { as } x_{1} \rightarrow-\infty .
$$

The above behaviour implies matching with the upstream response. To illustrate this, we take the pressure $p$ as an example. In the upper deck, the pressure $p$ is given by

$$
p=\delta R e^{\frac{3}{16}} \mu_{w}^{-\frac{3}{16}} T_{w}^{-\frac{9}{16}} M^{-\frac{3}{8}} A\left(x_{1}\right)\left[\bar{a}_{I} \mathrm{e}^{\mathrm{i} \hat{\gamma}_{\mathrm{TS}} \bar{y}}+\bar{a}_{R} \mathrm{e}^{-\mathrm{i} \hat{\gamma}_{\mathrm{TS}} \bar{y}}\right] \tilde{E}+\text { c.c. },
$$

where $\bar{a}_{I}$ and $\bar{a}_{R}$ are found from (4.25)-(4.26) as

$$
\bar{a}_{I, R}=\mp \frac{\hat{\alpha}_{\mathrm{TS}} \mathrm{e}^{\mp \mathrm{i} \hat{\gamma}_{\mathrm{TS}} \bar{y}_{s}}}{2 \lambda_{0} \hat{\gamma}_{\mathrm{TS}} \cos \left(\hat{\gamma}_{\mathrm{TS}} \bar{y}_{s}\right)} \int_{\eta_{0}}^{\infty} \operatorname{Ai}(\eta) \mathrm{d} \eta .
$$

Using (5.2), (4.36) and (2.42), we find that

$$
\bar{a}_{I, R} A\left(x_{1}\right) \rightarrow\left\{\frac{x_{0} J_{0}\left[\tan \left(\hat{\gamma}_{\mathrm{TS}} \bar{y}_{s}\right) \pm \mathrm{i}\right]}{b \cos \left(\hat{\gamma}_{\mathrm{TS}} \bar{y}_{s}\right)} \int_{\eta_{0}}^{\infty} \operatorname{Ai}(\eta) \mathrm{d} \eta\right\} x_{1}^{-1} \text { as } x_{1} \rightarrow-\infty .
$$

Comparison with (3.24) shows that

$$
R e^{\frac{3}{16}} \mu_{w}^{-\frac{3}{16}} T_{w}^{-\frac{9}{16}} M^{-\frac{3}{8}} A\left(x_{1}\right) \bar{a}_{I, R} \rightarrow a_{I, R} \quad \text { as } \quad x_{1} \rightarrow-\infty,
$$

which is precisely what is required for the matching of the pressure in the two stages.

\subsection{Coupling coefficients}

We now turn to the downstream limit, $x_{1} \rightarrow+\infty$. In this limit,

$$
A\left(x_{1}\right) \rightarrow A_{\infty} \mathrm{e}^{\varsigma x_{1}^{2} / 2}, \quad \text { with } \quad A_{\infty}=N \int_{-\infty}^{\infty} \mathrm{e}^{-\varsigma \xi^{2} / 2} \mathrm{~d} \xi=N \sqrt{2 \pi / \varsigma}
$$

In the post-resonance stage corresponding to $x_{1}=O\left(\bar{\epsilon}^{-1}\right)$, the amplitude of the excited T-S mode, $A \mathrm{e}^{\mathrm{i} \hat{\alpha}_{\mathrm{TS}} \bar{x}}$, is replaced by the usual WKBJ form

$$
A_{\infty} \exp \left\{\mathrm{i} R e^{\frac{3}{8}} \mu_{w}^{-\frac{3}{8}} T_{w}^{-\frac{9}{8}} M^{-\frac{3}{4}} \int_{x_{0}}^{x} \hat{\alpha}_{\mathrm{TS}}(x) \mathrm{d} x\right\},
$$

where the complex wavenumber $\hat{\alpha}_{\mathrm{TS}}(x)$ is determined by the local parallel stability theory. Clearly, the constant $A_{\infty}$ appears as the (scaled) initial amplitude.

In order to be precise, we use the maximum value of the streamwise velocity of the T-S wave as an indicator of its magnitude. First, inserting $\tilde{P}_{1}$ found from (4.25) into 
the spanwise momentum equation in (4.21), we may express the spanwise velocity as $\tilde{W}_{1}=\left(\mathrm{i} \mathrm{Ai}^{\prime}\left(\eta_{0}\right) / \hat{\beta}_{\mathrm{TS}}\right) L(\eta)$, where $L(\eta)$ is the solution to the boundary-value problem:

$$
L^{\prime \prime}-\eta L=1, \quad L\left(\eta_{0}\right)=0 ; \quad L \rightarrow 0 \quad \text { as } \eta \rightarrow \infty .
$$

From the continuity equation, the streamwise velocity is obtained and its maximum magnitude at the neutral point is given by (cf. Wu 1999)

$$
u_{T S}=\delta R e^{\frac{5}{16}} \mu_{w}^{-\frac{5}{16}} T_{w}^{\frac{1}{16}} M^{\frac{11}{8}} \hat{\alpha}_{\mathrm{TS}}^{-1} A_{\infty} U_{m},
$$

where

$$
U_{m}=\max _{\eta}\left|\int_{\eta_{0}}^{\eta} \operatorname{Ai}(\eta) \mathrm{d} \eta-\operatorname{Ai}^{\prime}\left(\eta_{0}\right) L(\eta)\right| .
$$

Following the practice in experiments, we normalize the dimensional frequency of the disturbance $\breve{\omega}$ as

$$
f=\breve{\omega} \breve{\nu}_{s} / \breve{U}_{s}^{2}
$$

For the rescalings (2.30), we find by using (4.28)-(4.29) that the neutral position, nondimensionalized by $\breve{\mu}_{s} / \breve{\rho}_{s} \breve{U}_{s}$, is given by

$$
\tilde{x}_{0} \equiv \frac{\breve{\rho}_{s} \breve{U}_{s} \breve{x}_{0}}{\breve{\mu}_{s}}=\mu_{w}^{-\frac{1}{3}} T_{w}^{-1} M^{-\frac{2}{3}} \lambda_{0}^{2}\left(f / \omega_{N}\right)^{-4 / 3} .
$$

The streamwise length scale $\breve{l}$ can be taken as $\breve{x}_{0}$ so that $x_{0}=1, \lambda_{0}=\chi$ and the Reynolds number $R e$ is actually $\tilde{x}_{0}$.

We define the coupling coefficient for the fast/slow acoustic waves respectively as

$$
C_{A}^{ \pm}=u_{T S} / \delta p_{I}^{ \pm}
$$

and it follows from (5.7), (4.36) and (5.11)-(5.12) that

$$
C_{A}^{ \pm}=\frac{2}{2-\bar{\gamma}} \sigma^{2} \mu_{w}^{-\frac{5}{12}} T_{w}^{-\frac{1}{4}} M^{\frac{19}{6}} f^{-\frac{5}{12}} \kappa
$$

where

$$
\kappa\left(\beta_{N}, y_{N}\right)=\sqrt{2 \pi} \chi^{13 / 8} \omega_{N}^{5 / 12} \sqrt{1-\left(\beta_{N} / \alpha_{N}\right)^{2}} \sec \left(\sqrt{\alpha_{N}^{2}-\beta_{N}^{2}} y_{N}\right) U_{m} /|a \sqrt{\varsigma}| .
$$

Similarly, we define the coupling coefficients for the vorticity and entropy waves respectively as

$$
C_{V} \equiv u_{T S} / \delta v_{I}, \quad C_{E} \equiv u_{T S} / \delta r_{I} .
$$

It is found that $C_{V}$ and $C_{E}$ scale with $f$ and $M$ as

$$
C_{V}=2 \sigma^{-1} \mu_{w}^{-\frac{5}{12}} T_{w}^{-\frac{1}{4}} M^{-\frac{5}{6}} f^{-\frac{5}{12}} \kappa, \quad C_{E}=\sigma^{2} \mu_{w}^{-\frac{5}{12}} T_{w}^{-\frac{1}{4}} M^{\frac{7}{6}} f^{-\frac{5}{12}} \kappa .
$$



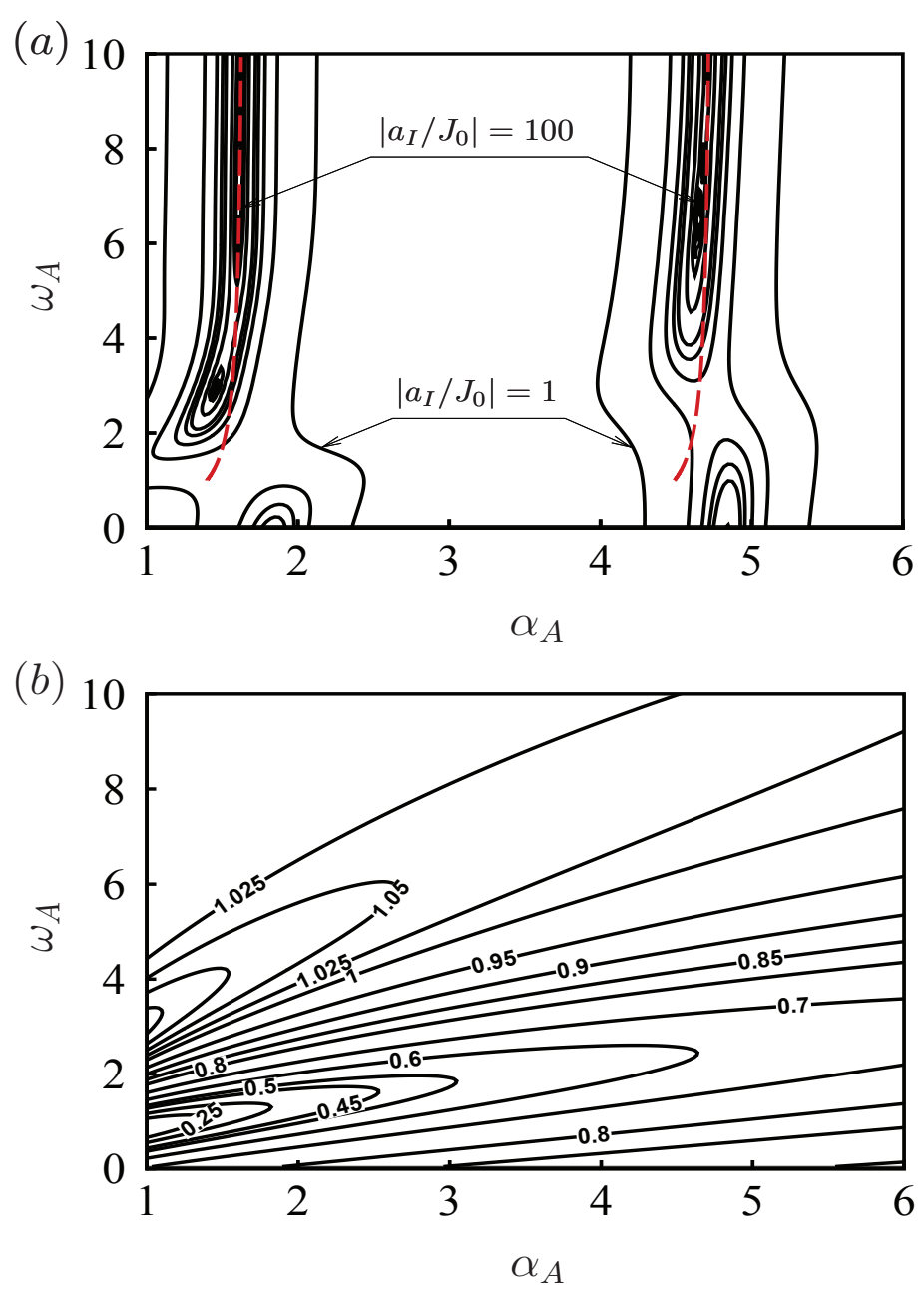

Figure 7: Contours of $\left|a_{I} / J_{0}\right|$ and $|\mathscr{R}|$ in the $\alpha_{A}-\omega_{A}$ plane for $y_{A}=1$ and $\beta_{A}=0.5$. The dashed lines in (a) represent the loci of (6.3) for $n=1,2$. 


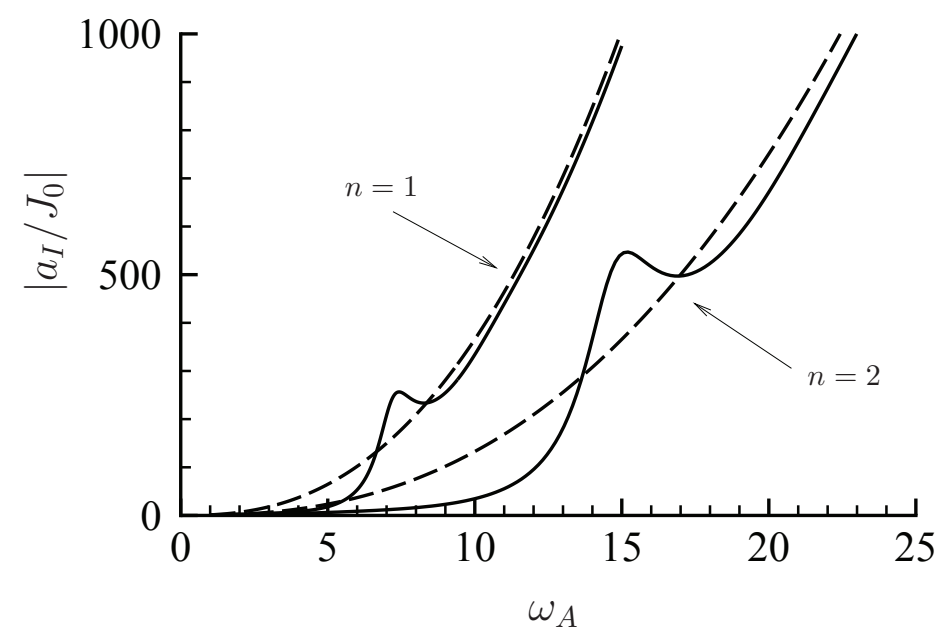

Figure 8: Peak values of $\left|a_{I} / J_{0}\right|$ at quasi-resonance. Solid lines: numerical solution calculated from (3.21); dashed lines: asymptotic approximation (6.2) for $\omega_{A} \gg 1$.

\section{Numerical results}

\subsection{Boundary-layer response in the pre-resonance region}

The boundary-layer responds to a broadband of free-stream disturbances, including to vortical and entropy disturbances since they generate acoustic waves downstream of the shock. We first consider the characteristics of the response at a fixed streamwise location $x$. The dependence on $x$ is through $\lambda$ with $x$ acting as a parameter, and it can be scaled out by setting

$$
\hat{\alpha}_{a}=\lambda^{5 / 4} \alpha_{A}, \quad \hat{\beta}_{a}=\lambda^{5 / 4} \beta_{A}, \quad \hat{\omega}_{a}=\lambda^{3 / 2} \omega_{A}, \quad \bar{y}_{s}=\lambda^{-5 / 4} y_{A} .
$$

As an illustration, calculations are performed for $y_{A}=1$ and $\beta_{A}=0.5$ with $\alpha_{A}$ and $\omega_{A}$ in the range $1 \leq \alpha_{A} \leq 6$ and $0<\omega_{A} \leq 10$. Figure 7 shows the contours of $\left|a_{I} / J_{0}\right|$ and $|\mathscr{R}|$; contours of $\left|a_{R} / J_{0}\right|$ look similar to those of $\left|a_{I} / J_{0}\right|$ and hence are not shown. The response is finite for all $\left(\alpha_{A}, \omega_{A}\right)$ except when $\left(\alpha_{A}, \omega_{A}\right)=(1.46,2.95)$ and $(4.65,6.40)$, which correspond to neutral modes 1 and 2 respectively. A striking feature is that there is an extremely strong response to a broadband of high-frequency free-stream disturbances within a narrow band of $\alpha_{A}$, represented by the two narrow and almost vertical strips in the figure. In order to explain this, an analysis of (3.21) is performed in the high-frequency limit $\hat{\omega}_{a} \gg 1$. For a general $\alpha_{A}$, both the numerator and the denominator $\Delta$ are of $O(1)$. However, the latter reduces to $O\left(\omega_{A}^{-5 / 2}\right)$ if $\alpha_{A}$ is sufficiently close to

$$
\alpha_{c}=\left[\left[\frac{(2 n-1) \pi}{2 y_{A}}\right]^{2}+\beta_{A}^{2}\right]^{1 / 2}(n=1,2, \ldots)
$$

while the numerator remains of $O(1)$ so that $a_{I, R} / J_{0}$ raises to $O\left(\omega_{A}^{5 / 2}\right)$. More precisely, the analysis shows that

$$
a_{I, R} / J_{0} \sim(-1)^{n} \frac{(2 n-1) \pi \mathrm{e}^{-\pi \mathrm{i} / 4}}{4 \alpha_{c}^{2} \beta_{A}^{2} y_{A}} \omega_{A}^{5 / 2},
$$



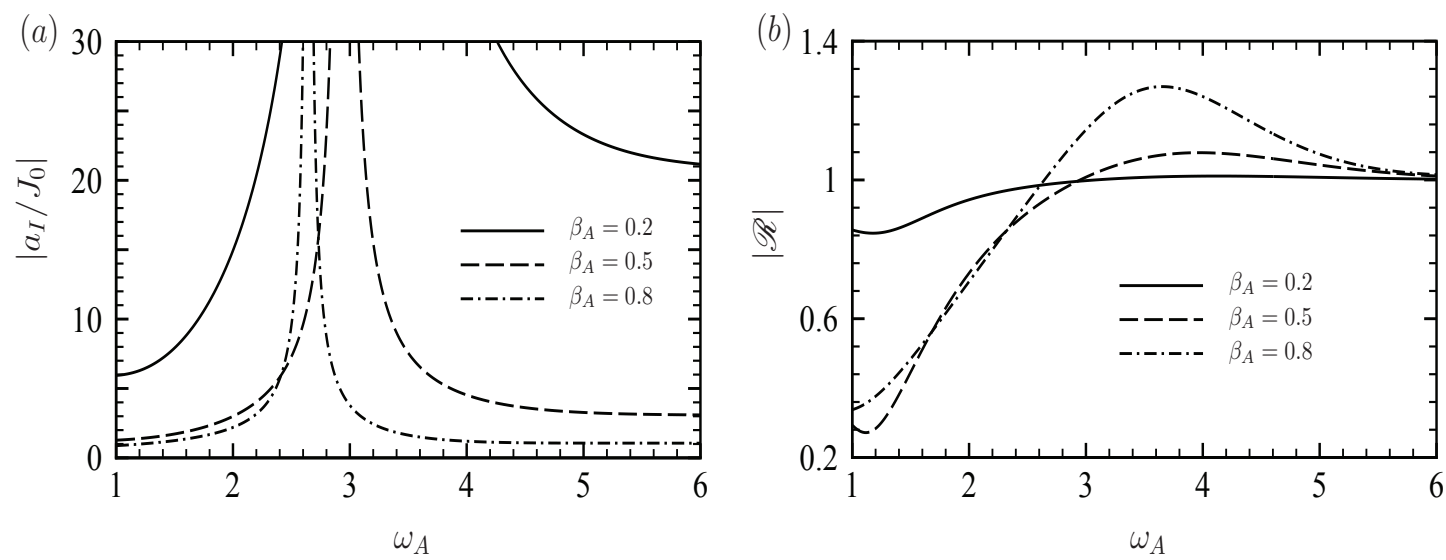

Figure 9: Variation of $\left|a_{I} / J_{0}\right|$ and $|\mathscr{R}|$ with $\omega_{A}$ for $\alpha_{A}$ being the wavenumber of mode 1.

when

$$
\alpha_{A}=\alpha_{c}-\left(\beta_{A}^{2} / y_{A}\right) \omega_{A}^{-1}-\frac{2 \beta_{A}^{6}}{(2 n-1)^{2} \pi^{2} \alpha_{c}} \omega_{A}^{-2} \text { and } \omega_{A} \gg 1 .
$$

The loci of (6.3) are marked by the dashed lines in figure 7(a). Figure 8 compares the numerical result with the asymptotic approximation (6.2). A good agreement is observed. The strong response is therefore due to the fact that the dispersion function $\Delta$ almost vanishes for $\alpha_{A}$ and $\omega_{A}$ specified by (6.3), and we will refer to this as quasi- or nearresonance (which differs from the exact resonance corresponding to $\Delta=0$ ). The quasiresonance is of broadband nature with respect to the frequency but highly selective with respect to the wavenumber. For each $\beta_{A}$, there exists a series of strips centred at the curves as specified by (6.3). In the presence of a broadband of free-stream disturbances, the boundary layer acts as a high-pass amplifier. Figure $7(b)$ indicates that for relatively low frequencies $|\mathscr{R}|<1$, that is, the boundary layer absorbs some of the incident acoustic wave energy. In contrast, for high-frequency waves, $|\mathscr{R}|>1$, implying that the wave reflected by the viscous boundary layer is stronger than the incident wave, a phenomenon referred to as over-reflection.

We are primarily interested in cases where an exact resonance with a T-S wave can take place, and thus in subsequent calculations for each $\beta_{A}$ the rescaled wavenumber $\alpha_{A}$ is taken to be that determined by the first relation in (4.29) for modes 1 and 2 . The computed $\left|a_{I} / J_{0}\right|$ and $|\mathscr{R}|$ as functions of $\omega_{A}$ are shown in figure 9 for mode $1 ;\left|a_{R} / J_{0}\right|$ is not shown as it resembles $\left|a_{I} / J_{0}\right|$. The response is finite for $\omega_{A} \neq \omega_{N}$, but becomes infinite as the frequency approaches the local neutral frequency $\omega_{N}$ of mode 1 , indicating an exact resonance between the sound wave and the neutral T-S mode. Figures $9(a)$ shows that $\left|a_{I} / J_{0}\right|$ is larger for smaller spanwise wavenumbers. Figure $9(b)$ indicates $|\mathscr{R}|<1$ for low frequencies and $|\mathscr{R}|>1$ for high frequencies as observed earlier. Interestingly, (3.23) indicates that

$$
\mathscr{R} \rightarrow \frac{\tan \left(\hat{\gamma}_{a} \bar{y}_{s}\right)-\mathrm{i}}{\tan \left(\hat{\gamma}_{a} \bar{y}_{s}\right)+\mathrm{i}} \text { as } \omega_{A} \rightarrow \omega_{N}
$$

so that $|\mathscr{R}| \rightarrow 1$. The parameter region of over-reflection, $\omega_{A}>\omega_{N}$, corresponds to the unstable regime above the neutral curve. The present consideration of reflection thus provides a physical interpretation of the shock-induced instability. A similar instability 

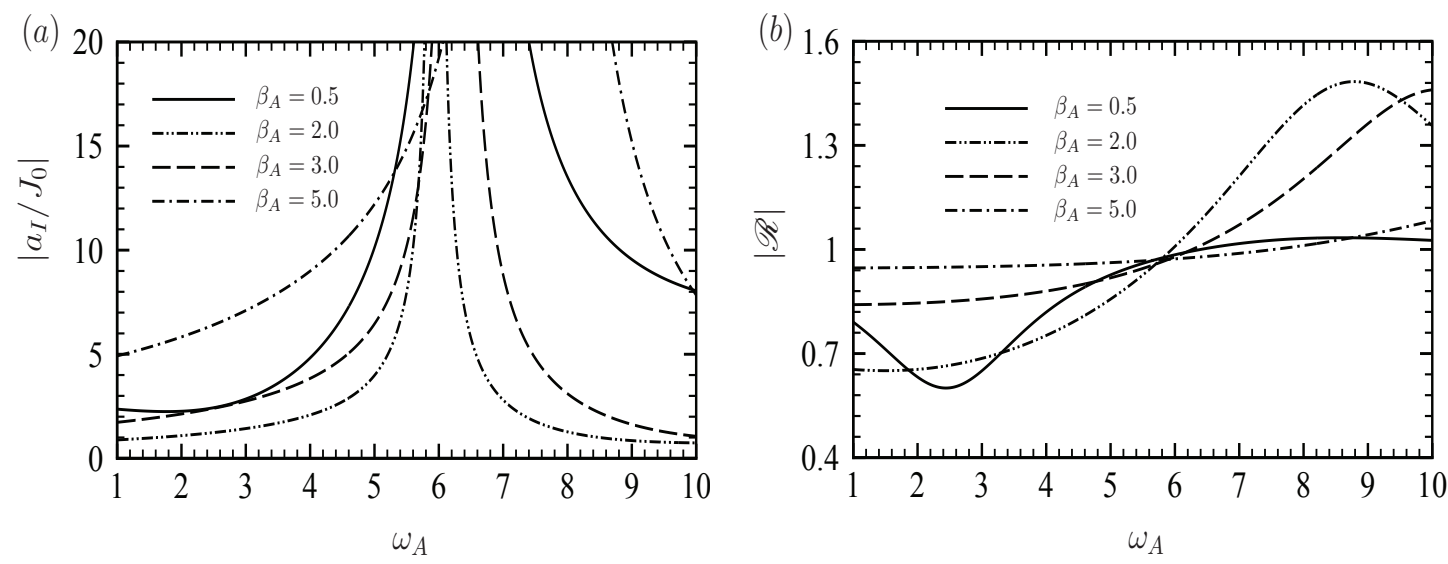

Figure 10: Variation of $\left|a_{I} / J_{0}\right|$ and $|\mathscr{R}|$ with $\omega_{A}$ for $\alpha_{A}$ being the wavenumber of mode 2 .

arises in the supersonic twin boundary layers developing along two parallel plates $(\mathrm{Wu}$ 2014).

Figures $10(a),(b)$ depict the variations of $\left|a_{I} / J_{0}\right|$ and $|\mathscr{R}|$ with the frequency $\omega_{A}$ for different spanwise wavenumbers $\beta_{A}$ with $\alpha_{A}$ being the wavenumber of mode 2 . The amplitudes $\left|a_{I} / J_{0}\right|$ and $\left|a_{R} / J_{0}\right|$ (not shown) first decrease and then increase as the spanwise wavenumber $\beta_{A}$ varies from 0.5 to 5.0 in both the low- and high-frequency regions. This may be associated with the non-monotonicity of the neutral curve of mode 2 (see figure $3)$. The reflection coefficient $|\mathscr{R}|$ exhibits a similar feature to that in figure $9(b):|\mathscr{R}|<1$ for low-frequency waves and $|\mathscr{R}|>1$ for high-frequency waves with $|\mathscr{R}|=1$ on the neutral curve, signaling that the onset of the instability corresponds to over-reflection.

Next, we consider the response at different streamwise locations for fixed physical frequencies $\hat{\omega}_{a}$ and wavenumbers $\hat{\alpha}_{a}$ and $\hat{\beta}_{a}$. The latter two are specified as

$$
\hat{\alpha}_{a}=\chi^{5 / 4} \alpha_{N}, \quad \hat{\beta}_{a}=\chi^{5 / 4} \beta_{N},
$$

where $\chi=0.332$, and $\alpha_{N}$ and $\beta_{N}$ are on the neutral curves of mode 1 or 2 . The calculations were performed for $\bar{y}_{s}=\chi^{-5 / 4} x$ with $0<x \leq 1.2$. For the case of $\beta_{N}=0.5$ and $\alpha_{N}$ being on the neutral curve of mode 1 , contours of $\left|a_{I} / J_{0}\right|$ are displayed in figure 11. A broadband of strong high-frequency disturbances appears at almost the same streamwise location, owing again to the quasi-resonance. A high-frequency analysis of (3.21) for fixed $\alpha_{N}$ and $\beta_{N}$ shows that the response raises to $O\left(\left(\hat{\omega}_{a} / \chi^{3 / 2}\right)^{5 / 2}\right)$, or more precisely,

$$
a_{I, R} / J_{0} \sim(-1)^{n} \frac{\left(\alpha_{N}^{2}-\beta_{N}^{2}\right)^{1 / 2} \mathrm{e}^{-\pi \mathrm{i} / 4}}{2 \alpha_{N}^{2} \beta_{N}^{2} \chi^{15 / 4}} \hat{\omega}_{a}^{5 / 2},
$$

when

$$
x=x_{c}-\frac{\alpha_{N} \beta_{N}^{2} x_{c}^{1 / 2} \chi^{3 / 2}}{\alpha_{N}^{2}-\beta_{N}^{2}} \hat{\omega}_{a}^{-1}-\frac{\alpha_{N}^{2} \beta_{N}^{4} \chi^{3}}{2\left(\alpha_{N}^{2}-\beta_{N}^{2}\right)^{2}} \hat{\omega}_{a}^{-2} \text { and } \hat{\omega}_{a} \gg 1,
$$

where

$$
x_{c}=\frac{(2 n-1) \pi}{2\left(\alpha_{N}^{2}-\beta_{N}^{2}\right)^{1 / 2}} \quad(n=1,2, \ldots) .
$$

The locus of (6.7), in the vicinity of which the response is the strongest, is indicated by the dashed line in figure 11. 


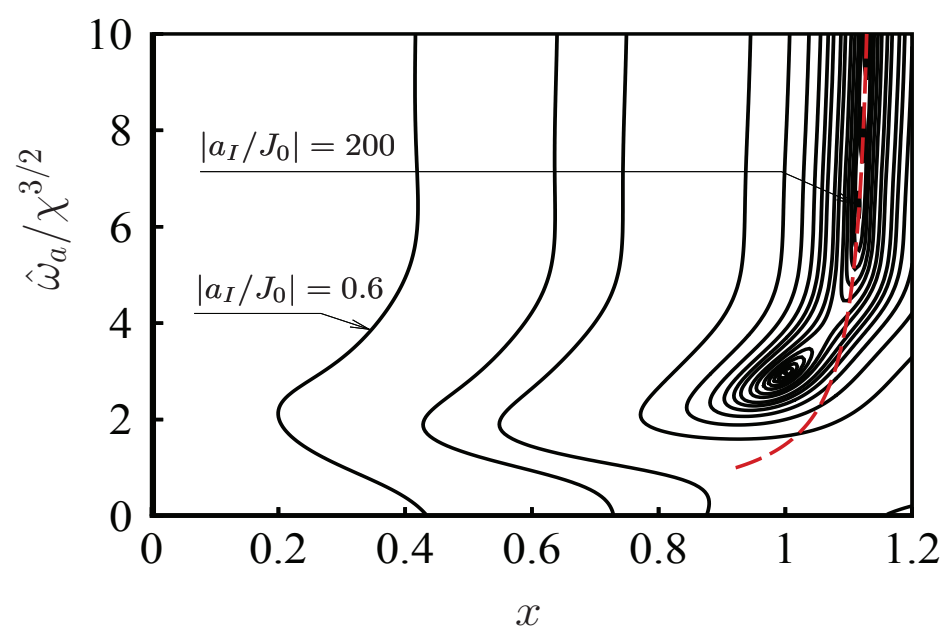

Figure 11: Contours of $\left|a_{I} / J_{0}\right|$ and $|\mathscr{R}|$ in the $x-\hat{\omega}_{a} / \chi^{3 / 2}$ plane for $\bar{y}_{s}=\chi^{-5 / 4} x$ and $\beta_{N}=0.5$. The dashed line represents the locus of (6.7).
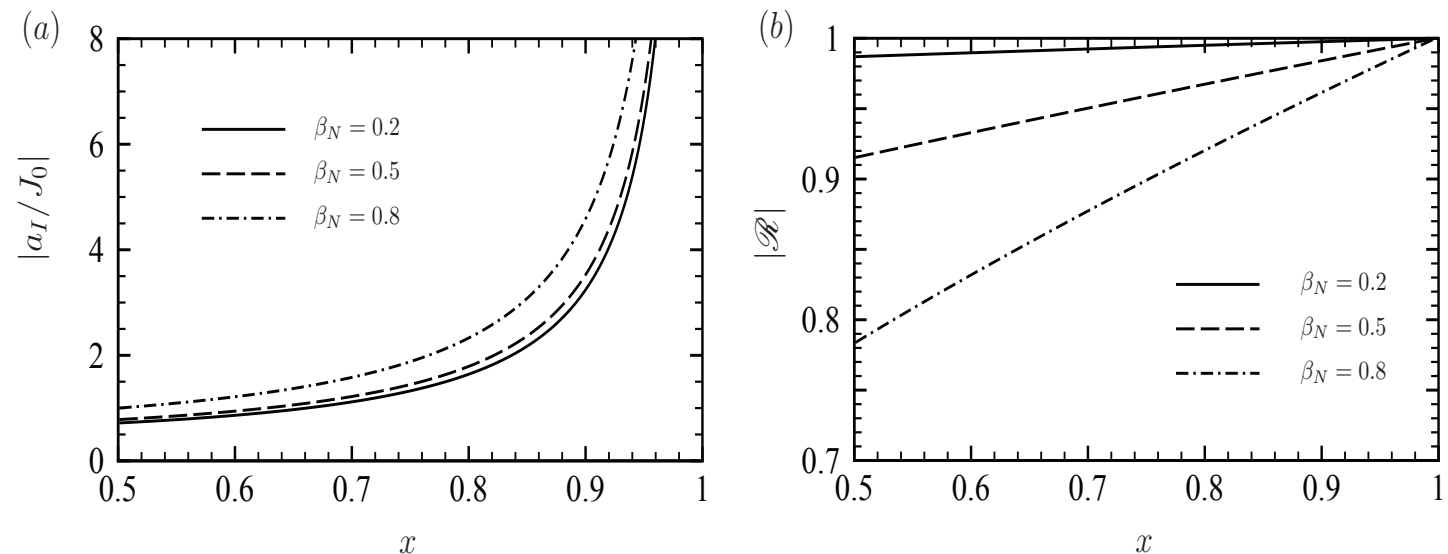

Figure 12: Variation of $\left|a_{I} / J_{0}\right|$ and $|\mathscr{R}|$ with $x$ for $\alpha_{N}$ being the wavenumber of mode 1.
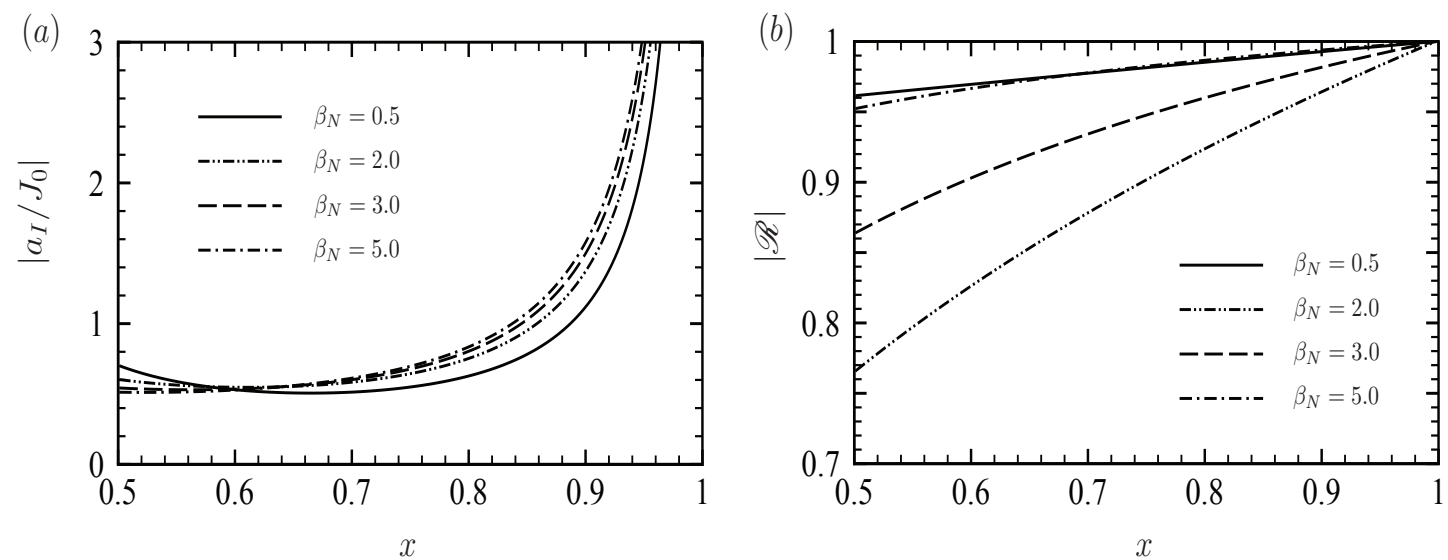

Figure 13: Variation of $\left|a_{I} / J_{0}\right|$ and $|\mathscr{R}|$ with $x$ for $\alpha_{N}$ being the wavenumber of mode 2. 
Exact resonance occurs (at $x=x_{0}=1$ ) when $\hat{\omega}_{a}=\chi^{3 / 2} \omega_{N}$. Figure 12 shows $\left|a_{I} / J_{0}\right|$ and $|\mathscr{R}|$ as functions of $x$ when the frequency is that of mode 1 . The variation pattern of $\left|a_{I} / J_{0}\right|$ and $|\mathscr{R}|$ is similar to that displayed in figure 9-10. The response is finite upstream of the neutral position $x_{0}=1$, but becomes infinite as $x \rightarrow x_{0}$. The response is stronger for disturbances with larger spanwise wavenumbers. Figure $12(b)$ indicates that $|\mathscr{R}|<1$ for $x<x_{0}=1$, and $|\mathscr{R}| \rightarrow 1$ when $x$ approaches the neutral point, downstream of which $|\mathscr{R}|>1$ (not shown), i.e. over-reflection takes place in the unstable region. Figures $13(a),(b)$ display the variation of $\left|a_{I} / J_{0}\right|$ and $|\mathscr{R}|$ with $x$ for different spanwise wavenumbers and the frequency being that of mode 2. Again, the amplitude of the response first decreases with the spanwise wavenumber and then increases, possibly due to the nonmonotonicity of the neutral curve of mode 2. The reflection coefficient $|\mathscr{R}|$ (figure $13(b)$ ) exhibits a similar feature to that of mode 1 .

The response of a supersonic boundary layer to incident slow acoustic waves was studied previously by Gaponov (1977), who used the linearized N-S equations but neglected the streamwise and spanwise diffusion as well as the effect of a shock. Over-reflection was observed, but an exact resonance with an eigen mode does not occur. Both phenomena may take place when acoustic waves interact with a supersonic vortex sheet (Ribner 1957), where the eigen modes are a compressible version of Kelvin-Helmholtz instability waves.

\subsection{Results for coupling coefficients}

In order to gain quantitative information about the efficiency of the receptivity, the dependence of $\kappa$ on the parameters is investigated. Figure 14 shows $\kappa$ for modes 1,2 and 3 , and for different shock locations. Mode-1 family stands out: the coupling coefficient is significantly larger than those for modes 2 and 3, and especially for modes in the region $\beta_{N}>\alpha_{N}$, where $\kappa$ is about 100 and 10 times larger for $y_{N}=0.2$ and 1 respectively. It may be worth noting that when $\beta_{N}>\alpha_{N}$, the induced acoustic perturbation downstream of the shock is not purely sinusoidal in the direction normal to the shock. Rather it consists of two components, among which one amplifies while the other attenuates exponentially. Despite this difference, the perturbation exhibits, in terms of its phase speed, the key characteristics of a slow acoustic wave. For small $\beta_{N}$, the value of $\kappa$ for mode 2 is larger than that for mode 3 , but there is a crossover when $\beta_{N}$ exceeds a critical value. As the shock position $y_{N}$ increases, the value of $\kappa$ decreases for all three modes, implying that the coupling is weaker when the shock becomes more distant to the wall.

Figure 14 indicates that $\kappa=O(1)$ for typical $O(1)$ values of $\beta_{N}$ and $y_{N}$. However, the factors multiplying $\kappa$ in $C_{A}^{ \pm}, C_{V}$ and $C_{E}$ are large and so are these coupling coefficients. A calculation performed for a wedge with a half angle of $\pi / 9$ (i.e. $\left.20^{\circ}\right), M_{u}=20(M \approx 14)$ and $R e=5.0 \times 10^{6}$ (for which $y_{N} \approx 0.2$ ). The coupling coefficients for mode 1 with $0<\beta_{N}<2$ are shown in figure 15. The result indicates extraordinarily efficiency, which is caused by the resonant nature of the receptivity mechanism. Among three types of free-stream disturbances, acoustic waves are most efficient due to the amplification effect of shock at large- $M$ limit as (A.40) indicates. Entropy fluctuations come the second and vortical perturbations are least efficient of the three by comparison. Specifically for $\beta_{N}=1.5$, it is found that for mode $1\left(\omega_{N}=6.1\right), \kappa=2.01$,

$$
C_{A}^{ \pm}=0.144 \times 10^{6} \kappa, \quad C_{V}=0.846 \times 10^{2} \kappa, \quad C_{E}=0.360 \times 10^{3} \kappa,
$$

and for mode $2\left(\omega_{N}=17.8\right), \kappa=0.43$,

$$
C_{A}^{ \pm}=0.092 \times 10^{6} \kappa, \quad C_{V}=0.542 \times 10^{2} \kappa, \quad C_{E}=0.230 \times 10^{3} \kappa .
$$



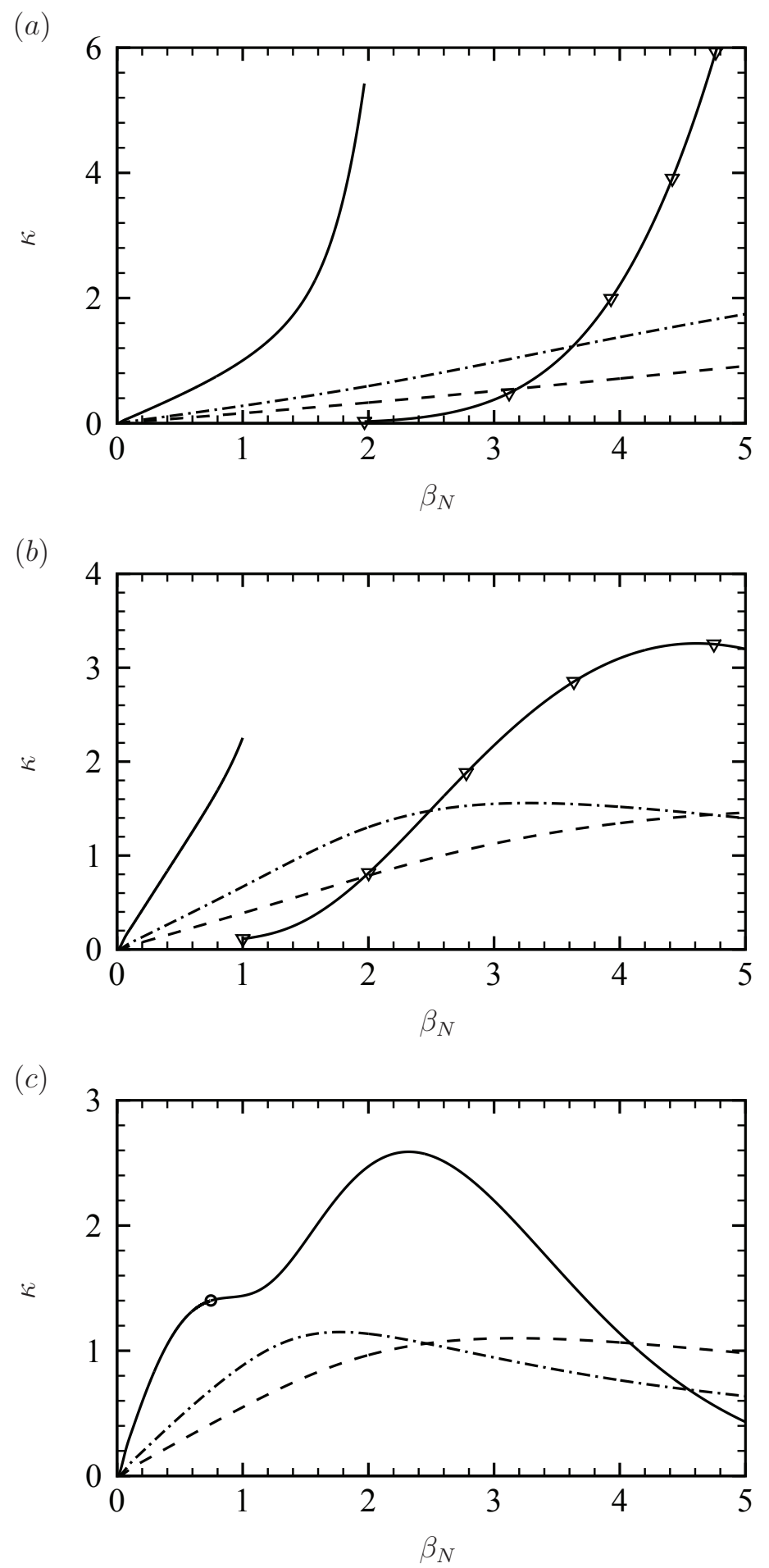

Figure 14: Variation of $\kappa$ with $\beta_{N}$ for $(a) y_{N}=0.2$, (b) $y_{N}=1$ and $(c) y_{N}=2$. Solid lines —-: mode 1 ; dash-dotted lines $-\cdot-\cdot-$ : mode 2 ; dashed lines --- : mode 3 . The curves $-\nabla-$ in $(a)$ and $(b)$ represent $\kappa / 200$ and $\kappa / 20$ respectively. 


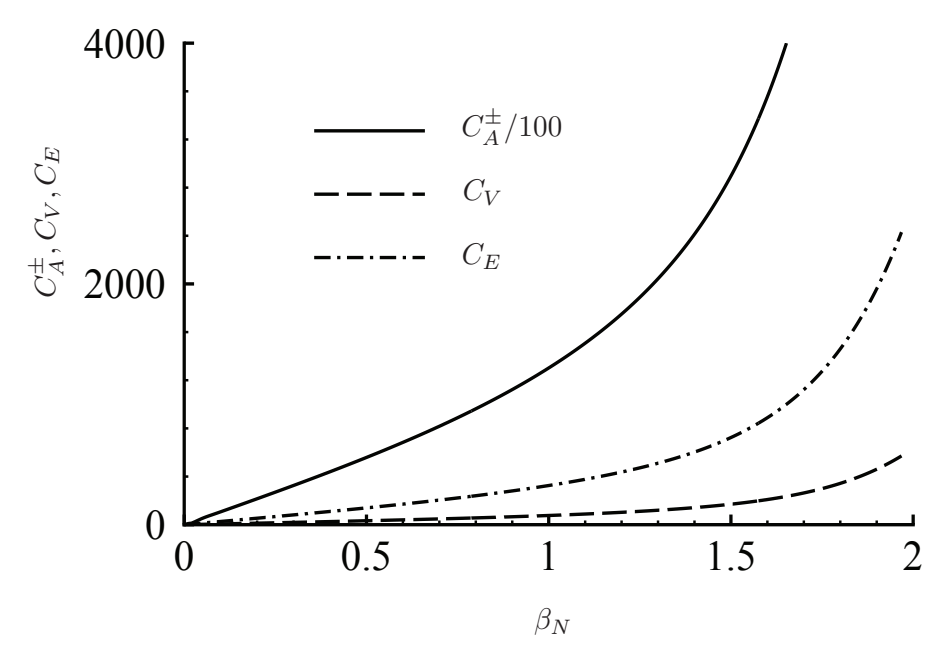

Figure 15: Variation of coupling coefficients $C_{A}^{ \pm}, C_{V}$ and $C_{E}$ with $\beta_{N}$ for a wedge with a half angle of $20^{\circ}, M_{u}=20$ and $R e=5.0 \times 10^{6}$, which corresponds to $y_{N} \approx 0.2$.

The absolute initial amplitude of the excited T-S mode depends on the level of fluctuations in the oncoming flow. The presence of a pressure fluctuation $\delta p_{I}=O\left(10^{-7}\right.$ ) (which corresponds to a velocity fluctuation of $\left.O\left(M_{u} \delta p_{I}\right)=O\left(10^{-6}\right)\right), \delta v_{I}=O\left(10^{-4}\right)$ and $\delta r_{I}=$ $O\left(10^{-4}\right)$ would generate T-S modes with initial amplitudes as large as a few per cent. The turbulence (noise) level in wind tunnels is usually higher than these by one or two orders of magnitude. Perturbations in flight conditions are thought to be weak, e.g. at cruise altitudes of civil aircrafts, the turbulence level, in terms of velocity fluctuations, is thought to be of $O\left(10^{-4}\right)$ with respect to a transonic flight speed. That translates to $O\left(10^{-5}\right)$ velocity fluctuation at $M_{u}=20$. On the other hand, density fluctuations of $O\left(10^{-5}\right)$ (which is independent of the flight speed) are very likely. Vortical or density perturbations of this level are capable of generating T-S waves with initial magnitudes in the range of $0.1-1 \%$, which is quite substantial. If velocity fluctuations of $O\left(10^{-5}\right)$ are primarily acoustic, the amplitude of T-S modes excited could be as large as 10\%, but of course in this case the validity of the present linear theory may be questioned.

Finally, it is worth noting that $\kappa \rightarrow 0$ as $\beta_{N} \rightarrow 0$. The present mechanism thus operates primarily for three-dimensional disturbances. Two-dimensional ones are probably more likely to excite instability modes through the leading edge mechanism of Fedorov \& Khokhlov (1991) and Fedorov (2003). However, if planar modes on the triple-deck scales are to be excited, new mechanisms must be sought.

\section{$7 \quad$ Summary and conclusions}

In this paper, the response and receptivity of the boundary layer over a wedge to freestream disturbances including acoustic, vortical and entropy perturbations were investigated theoretically in the limit of asymptotically large Reynolds and Mach numbers. We consider the case where the angle between the shock and the wedge is small so that the shock lies just at the outer edge of the boundary layer. In this case, there exist instability modes which assume a triple-deck structure and whose eigenfunctions are oscillatory in the upper deck. We focus on free-stream disturbances whose characteristic frequency and 
wavelength are also on the triple-deck scales. The boundary-layer response is viscous. Any of the three types of perturbations interacts with the shock to generate an acoustic perturbation, and thus free-stream vortical and entropy waves, like acoustic waves, can also drive velocity and temperature fluctuations in the boundary layer. A continuum of high-frequency disturbances within a narrow band of streamwise wavenumber was found to be in quasi-resonance with the eigen modes thereby generating remarkably strong boundary-layer response. This interesting phenomenon does not appear to have been reported before. We also monitored the behaviour of the reflection coefficient $\mathscr{R}$, defined as the ratio of the amplitude of the outgoing acoustic wave to that of the incoming wave, and noted that $|\mathscr{R}|<1$ upstream of the neutral point, and $|\mathscr{R}|>1$ downstream. The shock-induced instability can therefore be interpreted as over-reflection of acoustic waves by the boundary layer.

Among broadband three-dimensional free-stream perturbations, there exist components which generate slow acoustic waves whose streamwise and spanwise wavelengthes coincide with those of neutral T-S modes. The response to each of these components evolves through three stages, referred to as the pre-resonance, resonance and post-resonance stages. In the pre-resonance stage, the response, consisting of incoming and outgoing slow acoustic waves, is finite. The amplitudes of these two waves become unbounded as the neutral point of the corresponding T-S wave is approached. The disturbance enters the resonance stage in the vicinity of the neutral point, where the slow acoustic wave resonates with the T-S eigen mode. By using the matched asymptotic expansion and multiple scale methods simultaneously, an amplitude equation was derived to describe the evolution of the upstream response into a nearly neutral T-S wave, which becomes an unstable mode in the post-resonance stage further downstream. By matching between different stages, the initial amplitude of the T-S wave and appropriate coupling coefficients are defined. The present mechanism is new and unique in the following sense. On the one hand, unlike the leading-edge mechanisms (Goldstein 1983, Fedorov \& Khokhlov 1991, Fedorov 2003), the conversion into eigen modes takes place further downstream and locally, in the vicinity of the lower-branch neutral curve. Another difference is that the presence of a shock is crucial for the present mechanism, but it is not for that of Federov \& Khokhlov (1991). On the other hand, each of acoustic, vortical and entropy perturbations alone may generate a T-S wave without resorting to interaction among themselves (cf. Wu 1999) or with a surface roughness element (cf. Ruban 1984, Goldstein 1985). For these reasons the mechanism is also very efficient. Among three different types of perturbations, fast and slow acoustic waves turn out to be the most efficient, which is also the case in the leading-edge mechanism (Federov \& Khokhlov 2001). The coupling coefficient decreases as the distance of the shock to the wall increases, and is smaller for higher modes. The coupling coefficient vanishes when the oncoming disturbance is two-dimensional, that is, the mechanism operates primarily for three-dimensional disturbances. The practice relevance of the present mechanism and its importance relative to the existing ones depend on a number of factors including the intensity and spectrum of the free-stream disturbances, the Mach number, the location of shock as well as surface roughness. A comparative study in the multi-dimensional parameter space is needed but is beyond the scope of this paper.

DNS investigations by Ma \& Zhong (2003b, 2005) and by Balakumar (2003, 2005) have indicated too that planar acoustic, vortical and entropy disturbances alone can excite instability modes in a supersonic flat-plate boundary layer. These careful numerical studies have provided much important information about supersonic boundary-layer receptivity. The precise mechanisms however remain to be fully understood. The numerical results 
were primarily interpreted in terms of the leading-edge mechanism of Fedorov \& Khohklov (1991), which is most likely to be operating since the external disturbances are twodimensional. For three-dimensional disturbances, the present new mechanism may be relevant too, but DNS in three dimensions is necessary in order to shed further light on it. The two mechanisms can be differentiated since they operate, as was emphasized above, in different streamwise regions, and tend to excite modes of distinct characters. The mechanism of Fedorov \& Kholkhlov (1991) generates modes with $O(1)$ phase speeds and with spanwise wavenumbers $\beta<\sqrt{M^{2}-1} \alpha$. The present mechanism excite modes with phase speeds appreciably smaller than unity, among which high modes have spanwise wavenumbers $\beta>\sqrt{M^{2}-1} \alpha$, but the first mode is not subject to this restriction provided that $\beta \neq 0$.

Gaponov (1993) also considered excitation of instability waves in a supersonic flat-plate boundary layer by slow sound waves. However, without accounting for the presence of a shock, exact resonance with instability modes, which is instrumental in the mechanism described in the present paper, does not occur, that is, if a sound wave and an instability mode have the same frequency, their phase speeds would differ by a finite amount and so do their streamwise wavenumbers. The differences are fairly small numerically nevertheless, and so when a sound wave is refracted by the non-parallel boundary-layer flow, its phase speed/streamwise wavenumber may be tuned to match those of the instability mode so that the forcing resulting from refraction then excites the latter. This is clearly a different physical mechanism from what is described in the present paper, and it is also not as efficient. Mathematically, Gaponov (1993) used a heuristic non-asymptotic approach to calculate the coupling coefficient. Surprisingly, the strongest excitation was found to occur at the location of zero reflection.

It is known that at high Mach numbers, planar second Mack modes overall have the largest growth rates, but among first Mack modes the most amplified is three-dimensional. The present receptivity excites highly oblique waves, which may be considered as first Mack modes in their earlier stage (i.e. near the lower branch). When such a wave propagates downstream, its physical frequency and spanwise wavelength, remain fixed so that the non-dimensional frequency and spanwise wavenumber, normalized by the local boundarylayer thickness, will increase. The wave continues to amplify according to the result shown in figures 5 and 6 . It remains to be seen if some of these oblique modes would evolve into a predominantly inviscid regime and end up as nearly the most amplified modes. The answer to this question requires stability calculations to be performed in an extensive streamwise region well beyond the lower-branch triple-deck regime. On the other hand, in view of strong boundary-layer fluctuations generated through the quasi-resonance and the large amplitude of T-S waves excited through the exact resonance, the response and receptivity may well be nonlinear. Furthermore, the intense perturbations may even cause bypass transition. These issues merit further investigations.

The authors would like to thank Prof. P. Hall, Prof. A. I. Ruban, Dr. S. J. Cowley and the referees for helpful comments and suggestions. This work was supported by the NSFC (grants 11172204 and 11332007).

\section{A Shock conditions}

In this appendix, we consider the conditions which hold at the shock, the position of which is taken to be at $\eta=f\left(\xi, z, t ; x_{1}\right)$. The disturbance upstream is given by (2.11) along with 
(2.12) and (2.14)-(2.15), while that downstream is expressed by (2.18). In the presence of the small-amplitude disturbance, the shock is slightly perturbed from $\eta=0$ to

$$
\eta=f=\delta \tilde{\epsilon}^{-1}\left(\tilde{f}_{1}+\tilde{\epsilon} \tilde{f}_{2}+\cdots\right) \tilde{E}_{0} .
$$

The generalised shock conditions at $O\left(\delta \tilde{\epsilon}^{-1}\right)$ and $O(\delta)$ are derived by Qin \& Wu (2016) in a general setting. Those conditions are now specialised to (2.5a)-(2.5c).

\section{A.1 Shock condition of first order}

In the leading-order (i.e. $O\left(\delta \tilde{\epsilon}^{-1}\right)$ ) disturbance downstream the shock (see (2.17) and $(2.18))$, the acoustic part admits the solution

$$
\begin{gathered}
\tilde{p}_{1 a}=\left(\check{a}_{I} \mathrm{e}^{\mathrm{i} \nu_{1} \eta}+\check{a}_{R} \mathrm{e}^{\mathrm{i} \nu_{2} \eta}\right) \tilde{E}_{0}, \quad \tilde{r}_{1 a}=M^{2}\left(\check{a}_{I} \mathrm{e}^{\mathrm{i} \nu_{1} \eta}+\check{a}_{R} \mathrm{e}^{\mathrm{i} \nu_{2} \eta}\right) \tilde{E}_{0}, \\
\left(\tilde{u}_{1 a}, \tilde{v}_{1 a}, \tilde{w}_{1 a}\right)=\left[\frac{\check{a}_{I} \mathrm{e}^{\mathrm{i} \nu_{1} \eta}}{\Omega_{1} R}\left(\alpha, \nu_{1}, \beta\right)+\frac{\check{a}_{R} \mathrm{e}^{\mathrm{i} \nu_{2} \eta}}{\Omega_{2} R}\left(\alpha, \nu_{2}, \beta\right)\right] \tilde{E}_{0},
\end{gathered}
$$

where $\check{a}_{I}$ and $\check{a}_{R}$ are functions of $x_{1}$, and we have put $\Omega_{j}=\omega-\alpha U-\nu_{j} V(j=1,2)$.

The vorticity and entropy parts have the velocity and density fluctuations

$$
\left(\tilde{u}_{1 v}, \tilde{v}_{1 v}, \tilde{w}_{1 v}, \tilde{r}_{1 e}\right)=\left[\left(\check{u}_{s}, \check{v}_{s}, \check{w}_{s}, \check{r}_{s}\right)+O\left(\tilde{\epsilon}^{-2} \eta^{2} / V^{2}\right)\right] \tilde{E}_{0} \mathrm{e}^{\mathrm{i} \nu_{s} \eta}
$$

where $\nu_{s}=\nu_{s}\left(x_{1}\right)$ is the wavenumber in the $\eta$-direction. From the momentum and energy equations follows the dispersion relation

$$
\nu_{s}=\left(\omega-\alpha U+\tilde{\epsilon}^{-1} \mathrm{i} \check{a}_{I}^{\prime} / \check{a}_{I}\right) / V,
$$

while the continuity equation implies

$$
\alpha \check{u}_{s}+\beta \check{w}_{s}+\nu_{s} \check{v}_{s}=0 .
$$

The $O\left(\tilde{\epsilon}^{-2} \eta^{2} / V^{2}\right)$ term in (A.4) is of higher order for the disturbance on the triple-deck scales since $\eta=O\left(\tilde{\epsilon}^{2} M^{-1}\right)$, and does not affect the shock conditions at $\eta=0$ at any rate.

When applied to (2.5a)-(2.5c), the leading-order shock condition derived in Qin \& Wu (2016) can be written as (see also Cowley \& Hall 1990)

$$
\frac{K_{1}+K_{2} \nu_{1}}{\Omega_{1}} \check{a}_{I}+\frac{K_{1}+K_{2} \nu_{2}}{\Omega_{2}} \check{a}_{R}=0,
$$

where

$$
\begin{gathered}
K_{1}=-(\omega-\alpha U)\left\{(\gamma+1)(\epsilon-1)+4\left[(\omega-\alpha U)^{2}+\left(\alpha^{2}+\beta^{2}\right) V^{2}\right] / \mathscr{M}\right\}, \\
K_{2}=(\gamma+1)(1-\epsilon) \epsilon \mathscr{T} /\left(1+\epsilon^{2} \mathscr{T}^{2}\right)^{\frac{1}{2}}
\end{gathered}
$$

with

$$
\mathscr{M}=(\omega-\alpha U)^{2}+\left(\alpha^{2}+\beta^{2}\right) V \bar{V} .
$$

The solutions for the velocities $\left(\check{v}_{s}, \check{u}_{s}\right.$ and $\left.\check{w}_{s}\right)$ and the density $\check{r}_{s}$ can be written as

$$
\check{v}_{s}=\frac{\epsilon \mathscr{T}^{2}(\gamma+1)(1-\epsilon)^{2}(\omega-\alpha U)\left(\alpha^{2}+\beta^{2}\right)\left(\nu_{2}-\nu_{1}\right)}{\left(1+\epsilon^{2} \mathscr{T}^{2}\right)\left(K_{1}+K_{2} \nu_{2}\right) \Omega_{1} \mathscr{M}},
$$




$$
\begin{gathered}
\left(\check{u}_{s}, \check{w}_{s}\right)=\frac{\mathscr{T}(\gamma+1)(1-\epsilon)^{2}(\omega-\alpha U)^{2}\left(\nu_{2}-\nu_{1}\right)}{\left(1+\epsilon^{2} \mathscr{T}^{2}\right)^{\frac{1}{2}}\left(K_{1}+K_{2} \nu_{2}\right) \Omega_{1} \mathscr{M}}\left(\alpha \check{a}_{I}, \beta \check{a}_{I}\right), \\
\check{r}_{s}=-\frac{2\left(\gamma^{2}-1\right)(1-\epsilon)^{2}(\omega-\alpha U)\left[(\omega-\alpha U)^{2}+\left(\alpha^{2}+\beta^{2}\right) V^{2}\right]\left(\nu_{1}-\nu_{2}\right)}{V[\gamma+1-(\gamma-1) \epsilon]\left(K_{1}+K_{2} \nu_{2}\right) \Omega_{1} \mathscr{M}} \check{a}_{I} .
\end{gathered}
$$

In terms of $\check{u}_{s}$, the perturbation to the shock position is expressed as

$$
\tilde{f}_{1}=\frac{\mathrm{i}\left(1+\epsilon^{2} \mathscr{T}^{2}\right)^{\frac{1}{2}}}{\alpha(1-\epsilon) \mathscr{T}}\left[\check{u}_{s}+\frac{\alpha \check{a}_{I}}{\Omega_{1}}+\frac{\alpha \check{a}_{R}}{\Omega_{2}}\right] .
$$

For the disturbance on the triple-deck scales,

$$
(\alpha, \beta)=\tilde{\epsilon}^{-2}(\tilde{\alpha}, M \tilde{\beta}), \quad\left(\nu_{1}, \nu_{2}\right) \sim \tilde{\epsilon}^{-2} M \sqrt{\tilde{\alpha}^{2}-\tilde{\beta}^{2}}(1,-1) .
$$

From (2.13) and (2.29), we find that

$$
\nu_{0}^{ \pm} \sim \tilde{\epsilon}^{-2} \sigma^{-1}\left(\tilde{\alpha} \pm \sqrt{\frac{2-\bar{\gamma}}{2}} \tilde{\beta}\right) .
$$

Substitution of the scalings (2.28) and (A.14) into (A.8)-(A.9) shows that

$$
K_{1} \sim 2 \tilde{\epsilon}^{-2} \tilde{\alpha}\left(\tilde{\alpha}^{2}-\tilde{\beta}^{2}\right) /\left(\tilde{\alpha}^{2}+\tilde{\beta}^{2}\right), \quad K_{2} \sim 2 /\left(\sigma M^{2}\right) .
$$

Thus $\left|K_{1}\right| \gg\left|K_{2} \nu_{1,2}\right|$ and equation (A.7) reduces to (Cowley \& Hall 1990)

$$
\check{a}_{I}+\check{a}_{R}=0 .
$$

Substituting the scalings (2.28) and (A.14) into (A.10)-(A.12), we find that the vorticity and entropy modes associated with the T-S wave behave as (Cowley \& Hall 1990)

$$
\left(\check{u}_{s}, \check{v}_{s}, \check{w}_{s}, \check{r}_{s}\right) \sim 2 M \check{a}_{I}\left(\sigma \tilde{\alpha},-\tilde{\beta}^{2} / \tilde{\alpha}, \sigma M \tilde{\beta}, \sigma^{-1} \tilde{\alpha}\right) / \sqrt{\tilde{\alpha}^{2}-\tilde{\beta}^{2}} .
$$

\section{A.2 Shock condition of second order}

Consider now the $O(\delta)$ disturbance downstream the shock, which consists of the perturbations that are transmitted from, or generated by, the upstream perturbation as well as that arise from the modulation of the leading-order (i.e. $O(\delta / \bar{\epsilon})$ ) fluctuations associated with the T-S mode. The solution for the acoustic part is of the form

$$
\begin{gathered}
\tilde{p}_{2 a}=\left(\hat{a}_{I} \mathrm{e}^{\mathrm{i} \nu_{1} \eta}+\hat{a}_{R} \mathrm{e}^{\mathrm{i} \nu_{2} \eta}+\hat{p}_{2 a, p}\right) \tilde{E}_{0}, \\
\left(\tilde{u}_{2 a}, \tilde{v}_{2 a}, \tilde{w}_{2 a}\right)=\left[\frac{\hat{a}_{I} \mathrm{e}^{\mathrm{i} \nu_{1} \eta}}{\Omega_{1} R}\left(\alpha, \nu_{1}, \beta\right)+\frac{\hat{a}_{R} \mathrm{e}^{\mathrm{i} \nu_{2} \eta}}{\Omega_{2} R}\left(\alpha, \nu_{2}, \beta\right)+\left(\hat{u}_{2 a, p}, \hat{v}_{2 a, p}, \hat{w}_{2 a, p}\right)\right] \tilde{E}_{0}, \\
\tilde{r}_{2 a}=\left[M^{2}\left(\hat{a}_{I} \mathrm{e}^{\mathrm{i} \nu_{1} \eta}+\hat{a}_{R} \mathrm{e}^{\mathrm{i} \nu_{2} \eta}\right)+\hat{r}_{2 a, p}\right] \tilde{E}_{0},
\end{gathered}
$$

where $\hat{p}_{2 a, p}, \hat{u}_{2 a, p}, \hat{v}_{2 a, p}, \hat{w}_{2 a, p}$ and $\hat{r}_{2 a, p}$ stand for the particular solution. Their expressions are given in Qin \& Wu (2016), but are not needed for the present paper.

The second-order shock condition is

$$
\frac{K_{1}+K_{2} \nu_{1}}{\Omega_{1}} \hat{a}_{I}+\frac{K_{1}+K_{2} \nu_{2}}{\Omega_{2}} \hat{a}_{R}=\hat{J}_{P}^{+} p_{I}^{+}+\hat{J}_{P}^{-} p_{I}^{-}+\hat{J}_{V} v_{I}+\hat{J}_{R} r_{I}+\hat{J},
$$


where $\hat{J}_{P}^{ \pm}, \hat{J}_{V}$ and $\hat{J}_{R}$ represent the forcing of fast/slow acoustic, vorticity and entropy mode respectively, and their expressions for a general oblique shock are given in Qin \& $\mathrm{Wu}(2016)$. Inserting into them (2.5a)-(2.5c), we obtain

$$
\begin{gathered}
\hat{J}_{P}^{ \pm}=\hat{J}_{P, r}+\hat{J}_{P, u}^{ \pm}+\hat{J}_{P, v}^{ \pm}+\hat{J}_{P, p}, \\
\hat{J}_{V}=\frac{4 \epsilon \mathscr{T}(1-\epsilon)\left[(\omega-\alpha U)^{2}-\left(\alpha^{2}+\beta^{2}\right) V \bar{V}\right]}{\left(1+\epsilon^{2} \mathscr{T}^{2}\right)^{\frac{1}{2}} \mathscr{M}}, \\
\hat{J}_{R}=\frac{2(\epsilon-1) \mathscr{T}^{2}}{\left(1+\epsilon^{2} \mathscr{T}^{2}\right) \mathscr{M}}\left[(\omega-\alpha U)^{2}-\left(\alpha^{2}+\beta^{2}\right) V \bar{V}\right],
\end{gathered}
$$

with

$$
\begin{gathered}
\hat{J}_{P, r}=\frac{2 M_{u}^{2} \cos ^{2} \sigma(\epsilon-1) \mathscr{T}^{2}}{\cos ^{2} \phi\left(1+\epsilon^{2} \mathscr{T}^{2}\right) \mathscr{M}}\left[(\omega-\alpha U)^{2}-\left(\alpha^{2}+\beta^{2}\right) V \bar{V}\right], \\
\hat{J}_{P, u}^{ \pm}=-\frac{4 \epsilon^{2} \mathscr{T}^{2}\left(\alpha^{2}+\beta^{2}\right)(\omega-\alpha U)}{\left(1+\epsilon^{2} \mathscr{T}^{2}\right)\left(\omega-\alpha \bar{U}-\nu_{0}^{ \pm} \bar{V}\right) \bar{R} \mathscr{M}}, \\
\hat{J}_{P, v}^{ \pm}=\frac{4 \epsilon \mathscr{T}_{0}^{ \pm}}{\left(\omega-\alpha \bar{U}-\nu_{0}^{ \pm} \bar{V}\right) \bar{R}}\left[\frac{(\omega-\alpha U)^{2}-\left(\alpha^{2}+\beta^{2}\right)(1-\epsilon) V \bar{V}}{\left(1+\epsilon^{2} \mathscr{T}^{2}\right)^{\frac{1}{2}} \mathscr{M}}\right], \\
\hat{J}_{P, p}=(\gamma-1)(\epsilon-1)-\frac{4(\omega-\alpha U)^{2}}{\mathscr{M}} .
\end{gathered}
$$

It is interesting to note that although the upstream vorticity disturbance is characterized by two independent velocity components, $v_{I}$ and $u_{I}$, only $v_{I}$, the velocity normal to the shock, enters the shock condition to influence the disturbance downstream.

The last term $\hat{J}$ in (A.20) stands for the modulation of the leading-order disturbance, and it can be written as (Qin \& Wu 2016)

$$
\hat{J}=\hat{J}_{A}+\hat{J}_{F}+\hat{J}_{H}+\hat{J}_{\nu}
$$

among which

$$
\hat{J}_{A}=\left.\hat{J}_{A, u} \hat{u}_{2 a, p}\right|_{\eta=0}+\left.\hat{J}_{A, v} \hat{v}_{2 a, p}\right|_{\eta=0}+\left.\hat{J}_{A, w} \hat{w}_{2 a, p}\right|_{\eta=0},
$$

where

$$
\left.\begin{array}{l}
\left(\hat{J}_{A, u}, \hat{J}_{A, v}, \hat{J}_{A, w}\right)=\frac{4 \epsilon \mathscr{T}(\omega-\alpha U)}{\left(1+\epsilon^{2} \mathscr{T}^{2}\right) \mathscr{M}}\left(\epsilon \mathscr{T} \alpha,-(\omega-\alpha U)\left(1+\epsilon^{2} \mathscr{T}^{2}\right)^{\frac{1}{2}}, \epsilon \mathscr{T} \beta\right), \\
\left.\hat{u}_{2 a, p}\right|_{\eta=0}=\mathrm{i}\left(\mathcal{F}_{1}-\alpha V \mathcal{A}_{1} / \Omega_{1}\right) \frac{\check{a}_{I}^{\prime}}{\Omega_{1}}+\mathrm{i}\left(\mathcal{F}_{2}-\alpha V \mathcal{A}_{2} / \Omega_{2}\right) \frac{\check{a}_{R}^{\prime}}{\Omega_{2}}, \\
\left.\hat{v}_{2 a, p}\right|_{\eta=0}=\mathrm{i}\left[\mathcal{G}_{1}-(\omega-\alpha U) \mathcal{A}_{1} / \Omega_{1}\right] \frac{\check{a}_{I}^{\prime}}{\Omega_{1}}+\mathrm{i}\left[\mathcal{G}_{2}-(\omega-\alpha U) \mathcal{A}_{2} / \Omega_{2}\right] \frac{\check{a}_{R}^{\prime}}{\Omega_{2}}, \\
\left.\hat{w}_{2 a, p}\right|_{\eta=0}=\mathrm{i}\left(\mathcal{H}_{1}-\beta V \mathcal{A}_{1} / \Omega_{1}\right) \frac{\check{a}_{I}^{\prime}}{\Omega_{1}}+\mathrm{i}\left(\mathcal{H}_{2}-\beta V \mathcal{A}_{2} / \Omega_{2}\right) \frac{\check{a}_{R}^{\prime}}{\Omega_{2}},
\end{array}\right\}
$$

with

$$
\begin{gathered}
\mathcal{A}_{1,2}=-\tilde{\epsilon}^{-2} \frac{M^{2} \Omega_{1,2}+\alpha \cos \phi-\nu_{1,2} \sin \phi}{M^{2} V \Omega_{1,2}+\nu_{1,2}} \\
\left(\mathcal{F}_{1,2}, \mathcal{G}_{1,2}, \mathcal{H}_{1,2}\right)=-\tilde{\epsilon}^{-2}\left[\left(\frac{\alpha}{\Omega_{1,2}}+\cos \phi\right),\left(\frac{\nu_{1}}{\Omega_{1,2}}-\sin \phi\right), \frac{\beta}{\Omega_{1,2}}\right] .
\end{gathered}
$$


The remaining terms in (A.25) are

$$
\begin{gathered}
\hat{J}_{F}=-\tilde{\epsilon}^{-2} \frac{4 \epsilon^{2} \mathscr{T}(\epsilon-1)}{\left(1+\epsilon^{2} \mathscr{T}^{2}\right)^{2} \mathscr{M}}\left[\left(\alpha^{2}+\beta^{2}\right) U+\alpha(\omega-\alpha U)\right] \tilde{f}_{1}^{\prime}, \\
\hat{J}_{H}=-\tilde{\epsilon}^{-2} \frac{4 \mathrm{i} \epsilon^{2} \mathscr{T}^{2}(\omega-\alpha U)}{\left(1+\epsilon^{2} \mathscr{T}^{2}\right) \mathscr{M}}\left(-\cos \phi \check{u}_{s}^{\prime}+\sin \phi \check{v}_{s}^{\prime}\right), \\
\hat{J}_{\nu}=\tilde{\epsilon}^{-2} \frac{4 \epsilon \mathscr{T}^{2}\left(\alpha^{2}+\beta^{2}\right)(\omega-\alpha U)}{\left(1+\epsilon^{2} \mathscr{T}^{2}\right) \mathscr{M}^{2}}\left[\left(1-\frac{\epsilon \bar{\Omega}_{1}}{\Omega_{1}}\right) \check{a}_{I}+\left(1-\frac{\epsilon \bar{\Omega}_{2}}{\Omega_{2}}\right) \check{a}_{R}\right]\left(\mathrm{i} \check{a}_{I}^{\prime} / \check{a}_{I}\right),
\end{gathered}
$$

where we have put $\bar{\Omega}_{j}=\omega-\alpha U-\nu_{j} \bar{V}(j=1,2)$.

It is worth noting that if the modulation terms (i.e. $\hat{J}$ ) and the reflected wave component $\hat{a}_{R}$ are removed, the result (A.20) reduces to that given by McKenzie \& Westphal (1968) for a free-space shock.

We now simplify the shock condition (A.20) when the mean-flow property is subjec$\mathrm{t}$ to scaling constraints (2.28) and the perturbation is on the triple-deck scales (A.14). Substituting these scalings into (A.24a)-(A.24d) and (A.22)-(A.23), we find that

$$
\begin{gathered}
\hat{J}_{P}^{ \pm}=\hat{J}_{P, r}+\hat{J}_{P, u}^{ \pm}+\hat{J}_{P, v}^{ \pm}+\hat{J}_{P, p} \sim-\frac{4\left(\tilde{\alpha}^{2}-\tilde{\beta}^{2}\right)}{(2-\bar{\gamma})\left(\tilde{\alpha}^{2}+\tilde{\beta}^{2}\right)} \sigma^{2} M^{2}, \\
\hat{J}_{V} \sim \frac{4\left(\tilde{\alpha}^{2}-\tilde{\beta}^{2}\right)}{\tilde{\alpha}^{2}+\tilde{\beta}^{2}} \sigma^{-1} M^{-2}, \quad \hat{J}_{R} \sim-\frac{2\left(\tilde{\alpha}^{2}-\tilde{\beta}^{2}\right)}{\tilde{\alpha}^{2}+\tilde{\beta}^{2}} \sigma^{2} .
\end{gathered}
$$

In order to estimate $\hat{J}_{A}$, substitute (2.28) and (A.14) into (A.29)-(A.30), (A.27) and (A.28). It can be shown that

$$
\begin{gathered}
\left.\left(\hat{u}_{2 a, p}, \hat{v}_{2 a, p}, \hat{w}_{2 a, p}\right)\right|_{\eta=0} \sim \frac{\mathrm{i} \tilde{\beta}}{\tilde{\alpha}^{2}}\left[\frac{(\sigma M)^{-1} \tilde{\beta}}{\sqrt{\tilde{\alpha}^{2}-\tilde{\beta}^{2}}}\left(\check{a}_{I}^{\prime}-\check{a}_{R}^{\prime}\right), \frac{M \tilde{\beta}}{\sqrt{\tilde{\alpha}^{2}-\tilde{\beta}^{2}}}\left(\check{a}_{I}^{\prime}-\check{a}_{R}^{\prime}\right),-M\left(\check{a}_{I}^{\prime}+\check{a}_{R}^{\prime}\right)\right], \\
\left(\hat{J}_{A, u}, \hat{J}_{A, v}, \hat{J}_{A, w}\right) \sim-\frac{4 \tilde{\alpha}^{2}}{\tilde{\alpha}^{2}+\tilde{\beta}^{2}}\left(\sigma^{-2} M^{-4}, \sigma^{-1} M^{-2},(\tilde{\beta} / \tilde{\alpha}) \sigma^{-2} M^{-3}\right) .
\end{gathered}
$$

Inserting the above estimates into (A.26) shows that

$$
\left.\hat{J}_{A} \sim \hat{J}_{A, v} \hat{v}_{2 a, p}\right|_{\eta=0} \sim-\frac{4 \mathrm{i} \tilde{\beta}^{2}\left(\check{a}_{I}^{\prime}-\check{a}_{R}^{\prime}\right)}{\left(\tilde{\alpha}^{2}+\tilde{\beta}^{2}\right) \sqrt{\tilde{\alpha}^{2}-\tilde{\beta}^{2}}} \sigma^{-1} M^{-1} .
$$

Similarly, by considering (A.31) with (A.13), (A.32) and (A.33), we find that

$$
\begin{gathered}
\hat{J}_{F} \sim \frac{4 \mathrm{i} \tilde{\beta}^{2} \check{u}_{s}^{\prime}}{\tilde{\alpha}\left(\tilde{\alpha}^{2}+\tilde{\beta}^{2}\right)} \sigma^{-2} M^{-2}, \quad \hat{J}_{H} \sim-\frac{4 \mathrm{i} \tilde{\alpha} \check{u}_{s}^{\prime}}{\tilde{\alpha}^{2}+\tilde{\beta}^{2}} \sigma^{-2} M^{-4}, \\
\hat{J}_{\nu} \sim-\frac{4 \mathrm{i} \tilde{\alpha} \tilde{\beta}^{2} \check{a}_{I}^{\prime}}{\left(\tilde{\alpha}^{2}+\tilde{\beta}^{2}\right)^{2} \check{a}_{I}}\left(\check{a}_{I}+\check{a}_{R}\right),
\end{gathered}
$$

where it should be noted that the order of magnitude of $\check{u}_{s}$ is given by (A.18), and

$$
\check{a}_{I}+\check{a}_{R} \sim-4 \tilde{\alpha} \check{a}_{I}(\sigma M)^{-1} / \sqrt{\tilde{\alpha}^{2}-\tilde{\beta}^{2}},
$$

as can be deduced by inserting (2.28) and (A.14) into (A.7).

The general result (A.20) is now specified to three typical cases. 
- Case (1): only a fast or slow acoustic wave is present in the oncoming flow, $p_{I}^{ \pm} \neq 0$, $v_{I}=r_{I}=0$. The dominant balance in (A.20) indicates that $\hat{a}_{I}$ and $\hat{a}_{R}$ are $O\left(\sigma^{2} M^{2}\right)$, while (4.35) implies that the amplitude of T-S wave $A\left(x_{1}\right)$ is $O\left(\sigma^{2} M^{2}\right)$. Since $\hat{J}_{A}$, $\hat{J}_{F}, \hat{J}_{H}, \hat{J}_{\nu}$, are proportional to $A^{\prime}\left(x_{1}\right)$, we find that $\hat{J}_{A}, \hat{J}_{F}, \hat{J}_{\nu} \sim O(\sigma M)$ and $\hat{J}_{H} \sim O\left(\sigma M^{-1}\right)$, all much smaller than $\hat{J}_{P}^{ \pm}$. It follows that (A.20) simplifies to

$$
\hat{a}_{I}+\hat{a}_{R}=\frac{2}{2-\bar{\gamma}} \sigma^{2} M^{2} p_{I}^{ \pm} .
$$

The result indicates that the acoustic disturbance is, after propagating through the shock, amplified by a factor of $(\sigma M)^{2}$, as is known in the case of a free-space shock.

- Case (2): only a vorticity wave is present in the incoming flow, i.e. $v_{I} \neq 0, p_{I}^{+}=$ $p_{I}^{-}=r_{I}=0$. In this case $\hat{a}_{I}$ and $\hat{a}_{R}$ are $O\left(\sigma^{-1} M^{-2}\right)$, and the amplitude of T-S wave $A\left(x_{1}\right)$ is $O\left(\sigma^{-1} M^{-2}\right)$ according to (4.35). It follows that $\hat{J}_{A}, \hat{J}_{F}, \hat{J}_{\nu} \sim O\left(\sigma^{-2} M^{-3}\right)$ and $\hat{J}_{H} \sim O\left(\sigma^{-2} M^{-5}\right)$, all much smaller than $\hat{J}_{V}$ so that (A.20) becomes

$$
\hat{a}_{I}+\hat{a}_{R}=-2 \sigma^{-1} M^{-2} v_{I} .
$$

- Case (3): only an entropy wave is present in the oncoming flow, i.e. $r_{I} \neq 0, p_{I}^{+}=$ $p_{I}^{-}=v_{I}=0$. Balancing the left-hand side of (A.20) and $\hat{J}_{R}$ indicates that $\hat{a}_{I}$ and $\hat{a}_{R}$ are $O\left(\sigma^{2}\right)$. The amplitude of T-S wave $A\left(x_{1}\right)$ is $O\left(\sigma^{2}\right)$ as can be deduced from (4.35) and hence $\hat{J}_{A}, \hat{J}_{F}, \hat{J}_{\nu} \sim O\left(\sigma M^{-1}\right)$ and $\hat{J}_{H} \sim O\left(\sigma M^{-3}\right)$, which are much smaller than $\hat{J}_{R}$. Equation (A.20) thus reduces to

$$
\hat{a}_{I}+\hat{a}_{R}=\sigma^{2} r_{I}
$$

In summary, for all three cases above, the shock condition can be written as

$$
\hat{a}_{I}+\hat{a}_{R}=J_{0},
$$

where

$$
J_{0}= \begin{cases}\frac{2}{2-\bar{\gamma}} \sigma^{2} M^{2} p_{I}^{ \pm}, & \text {for fast/slow acoustic wave } \\ -2 \sigma^{-1} M^{-2} v_{I}, & \text { for vorticity wave } \\ \sigma^{2} r_{I}, & \text { for entropy wave. }\end{cases}
$$

Note that for disturbances on the triple-deck scales, the terms representing modulation in the general shock condition (A.20) are all of higher order and negligible.

In the pre-resonance region, the leading order (i.e. $O(\delta / \tilde{\epsilon})$ ) term representing the T-S wave signature is absent, i.e. $\check{a}_{I}=\check{a}_{R}=0$, and hence the general shock condition is (A.20) provided that we set $\hat{J}_{A}=\hat{J}_{F}=\hat{J}_{H}=\hat{J}_{\nu}=0$. After inserting the scalings (2.28) and (A.14), we obtain the same shock condition as (A.43).

\section{References}

[1] Balakumar, P. 2003 Transition in a supersonic boundary layer due to roughness and acoustic disturbances. AIAA Paper 2003-3589.

[2] Balakumar, P. 2005 Transition in a supersonic boundary layer due to acoustic disturbances. AIAA Paper 2005-0096. 
[3] Balakumar, P. 2009 Receptivity of a supersonic boundary layer to acoustic disturbances. AIAA J. 47 (5), 1069-1078.

[4] Balakumar, P. \& Kegerise, M. A. 2010 Receptivity of hypersonic boundary layers over straight and flared cones. AIAA Paper 2010-1545.

[5] Blackby, N., Cowley, S. J. \& Hall, P. 1993 On the instability of hypersonic flow past a flat plate. J. Fluid Mech. 247, 369-416.

[6] Chang, C. L., Malik, M. R. \& Hussaini, M. Y. 1990 Effects of shock on the stability of hypersonic boundary layers. AIAA Paper 1990-1448.

[7] Cowley, S. J. \& Hall, P. 1990 On the instability of hypersonic flow past a wedge. J. Fluid Mech. 214, 17-42.

[8] Dietz, A. J. 1999 Local boundary-layer receptivity to a convected free-stream disturbance. J. Fluid Mech. 378, 291-317.

[9] Dong, M. \& Wu, X. 2013 On continuous spectra of Orr-Sommerfeld/Squire equations and entrainment of free-stream vortical disturbances. J. Fluid Mech. 732, 616-659.

[10] Duck, P. W., Ruban, A. I. \& Zhikharev, C. N. 1996 Generation of Tollmien-Schlichting waves by free-stream turbulence. J. Fluid Mech. 312, 341-371.

[11] Duck, P. W., Lasseigne, D. G. \& Hussaini, M. Y. 1995 On the interaction between the shock wave attached to a wedge and freestream disturbances. Theor. Comput. Fluid Dyn. 7 (2), 119-139.

[12] Duck, P. W., Lasseigne, D. G. \& Hussaini, M. Y. 1997 The effect of three-dimensional freestream disturbances on the supersonic flow past a wedge. Phys. Fluids 9 (2), 456467.

[13] Egorov, I. V., Fedorov, A. V. \& Nechaev, A. V. 2004 Recptivity of supersonic boundary layers on a blunt plate to acoustic disturbances. AIAA Paper 2004-0249.

[14] Fedorov, A. V. 2003 Receptivity of a high-speed boundary layer to acoustic disturbances. J. Fluid Mech. 491, 101-129.

[15] Fedorov, A. V. 2011 Transition and stability of high-speed boundary layers. Annu. Rev. Fluid Mech. 43, 79-95.

[16] Fedorov, A. V. \& Khokhlov, A. P. 1991 Excitation of unstable modes in a supersonic boundary layer by acoustic waves. Fluid Dyn. 26 (4), 531-537.

[17] Fedorov, A. V. \& Khokhlov, A. P. 2001 Prehistory of instability in a hypersonic boundary layer. Theor. Comput. Fluid Dyn. 14 (6), 359-375.

[18] Gaponov, S. A. 1977 Interaction between a supersonic boundary layer and acoustic disturbances. Fluid Dyn. 12 (6), 858-862.

[19] Gaponov, S. A. 1993 Excitation of instability waves in the supersonic boundary layer by sound. In IUTAM Symposium on Nonlinear Instability of Nonparallel Flows, New York, USA (ed. S. P. Lin, W. R. C. Phillips \& D. T. Valentine). Springer-Verlag. 
[20] Goldstein, M. E. 1983 The evolution of Tollmien-Schlichting waves near a leading edge. J. Fluid Mech. 127, 59-81.

[21] Goldstein, M. E. 1985 Scattering of acoustic waves into Tollmien-Schlichting waves by small streamwise variations in surface geometry. J. Fluid Mech. 154, 509-529.

[22] Graziosi, P. \& Brown, G. 2002 Experiments on stability and transition at Mach 3. J. Fluid Mech. 474, 83-124.

[23] Hayes, W. D. \& Probstein, R. F. 2004 Hypersonic Inviscid Flow. Dover.

[24] Kendall, J. M. 1975 Wind tunnel experiments relating to supersonic and hypersonic boundary-layer transition. AIAA J. 13 (3), 290-299.

[25] King, R. A. 1992 Three-dimensional boundary-layer transition on a cone at Mach 3.5. Exp. Fluids 13, 305-314.

[26] Kovasznay, L. S. G. 1953 Turbulence in supersonic flow. J. Aero. Sci. 20, 657-674.

[27] Laufer, J. 1954 Fatcors affecting transition Reynolds numbers on models in supersonic wind tunnels. J. Aero. Sci. 21, 497-498.

[28] Laufer, J. 1960 Aerodynamic noise in supersonic wind tunnels. J. Aero. Sci. 28, 685-692.

[29] Ma, Y. \& Zhong, X. 2003a Receptivity of a supersonic boundary layer over a flat plate. Part 1: wave structures and interactions. J. Fluid Mech. 488, 31-78.

[30] Ma, Y. \& Zhong, X. 2003b Receptivity of a supersonic boundary layer over a flat plate. Part 2: receptivity to freestream sound. J. Fluid Mech. 488, 79-121.

[31] Ma, Y. \& Zhong, X. 2005 Receptivity of a supersonic boundary layer over a flat plate. Part 3: effects of different types of free-stream disturbances. J. Fluid Mech. 532, 63-109.

[32] Mack, L. M. 1984 Boundary-layer linear stability theory. AGARD Rep. 709.

[33] Maslov, A. A., Mironov, S. G., Kudryavtsev, A. N., Poplavskaya, T. V. \& Tsyryulnokov, I. S. 2010 Wave processes in a viscous shock layer and control of fluctuations. J. Fluid Mech. 60, 81-118.

[34] Maslov, A. A., Shiplyuk, A. N., Sidorenko, A. A. \& Arnal, D. 2001 Leading-edge receptivity of a hypersonic boundary layer on a flat plate. J. Fluid Mech. 426, 73-94.

[35] McKenzie, J. F. \& Westphal, K. O. 1968 Interaction of linear waves with oblique shock waves. Phys. Fluids 11, 2350-2362.

[36] Morkovin, M. V. 1969 Critical evaluation of transition from laminar to turbulent shear layers with emphasis on hypersonically travelling bodies. Tech. Rep. AFFDLTR, 68-149, US Air Force Flight Dynamics Laboratory, Wright Patterson Air Force Base, Ohio.

[37] Patel, S. R. 1971 Supersonic boundary-layer transition: effects of roughness and freestream disturbances. AIAA J. 9 (5), 797-803. 
[38] Petrov, G. V. 1984 Stability of thin viscous shock layer on a wedge in hypersonic flow of a perfect gas. In IUTAM Symposium on Laminar-Turbulent Transition, Novosibirsk, USSR (ed. V. V. Kozlov). Springer-Verlag.

[39] Qin, F. \& Wu, X. 2016 Interaction of three-dimensional disturbances with an oblique shock in the presence of a downstream boundary: generalized shock conditions. In preparation.

[40] Reshotko, E. 1976 Boundary layer stability and transition. Annu. Rev. Fluid Mech. 8, 311-349.

[41] Reshotko, E. 2008 Transition issues for atmospheric entry. J. Spacecr. Rockets 45 (2), 161-164.

[42] Ribner, H. S. 1957 Reflection, transmission, and amplification of sound by a moving medium. J. Acoust. Soc. Am. 29 (4), 435-441.

[43] Ruban, A. I. 1984 On Tollmien-Schlichting wave generation by sound. Izv. Akad. Nauk SSSR, Mekh. Zhidk. Gaza 5, 44-52.

[44] Schneider, S. P. 2001 Effect of high-speed tunnel noise on laminar-turbulent transition. J. Spacecr. Rockets 38 (3), 323-333.

[45] Seddougui, S. O. \& Bassom, A. P. 1994 Nonlinear instability of viscous modes in hypersonic flow past a wedge. Q. J. Mech. App. Maths. 47 (4) 557-582.

[46] Semionov, N. V. \& Kosinov, A. D. 1999 Experimental study of supersonic boundary layer receptivity in controlled conditions. In IUTAM Symposium on Laminar-Turbulent Transition, Arizona, USA (ed. H. F. Fasel \& W. S. Saric). Springer-Verlag.

[47] Semionov, N. V. \& Kosinov, A. D. 2008 An experimental study of receptivity of supersonic boundary layer on a blunted plate. Intl. J. Mech. 2 (3), 87-95.

[48] Smith, F. T. 1989 On the first-mode instability in subsonic, supersonic or hypersonic boundary layers. J. Fluid Mech. 198, 127-153.

[49] Smith, F. T. \& Brown, S. N. 1990 The inviscid instability of a Blasius boundary layer at large values of the Mach number. J. Fluid Mech. 219, 499-518.

[50] Wu, X. 1999 Generation of Tollmien-Schlichting waves by convecting gusts interacting with sound. J. Fluid Mech. 397, 285-316.

[51] Wu, X. 2001a On local boundary-layer receptivity to vortical disturbances in the free stream. J. Fluid Mech. 449, 373-393.

[52] Wu, X. 2001b Receptivity of boundary layers with distributed roughness to vortical and acoustic disturbances: a second-order asymptotic theory and comparison with experiments. J. Fluid Mech. 431, 91-133.

[53] Wu, X. 2014 On the role of acoustic feedback in boundary-layer instability. Phil. Trans. R. Soc. Lond. 372 (2020), 20130347.

[54] Zhong, X. 2001 Leading-edge receptivity to free-stream disturbance waves for hypersonic flow over a parabola. J. Fluid Mech. 441, 315-367. 
[55] Zhong, X. \& Ma, Y. 2006 Boundary-layer receptivity of Mach 7.99 flow over a blunt cone to free-stream acoustic waves. J. Fluid Mech. 556, 55-103.

[56] Zhong, X. \& Wang, X. 2012 Direct numerical simulation on the receptivity, instability and transition of hypersonic boundary layers. Annu. Rev. Fluid Mech. 44, 527-561. 